\title{
Identifying and exploring biohydrogenating rumen bacteria with emphasis on pathways including trans-10 intermediates
}

\author{
Lore Dewanckele $^{1 \dagger}$, Jeyamalar Jeyanathan ${ }^{1 \dagger}$, Bruno Vlaeminck $^{1,2}$ and Veerle Fievez ${ }^{1 *}$ (D)
}

\begin{abstract}
Background: Bacteria involved in ruminal formation of trans-10 intermediates are unclear. Therefore, this study aimed at identifying rumen bacteria that produce trans-10 intermediates from 18-carbon unsaturated fatty acids.

Results: Pure cultures of 28 rumen bacterial species were incubated individually in the presence of $40 \mu \mathrm{g} / \mathrm{mL} 18: 3 \mathrm{n}$ 3, 18:2n-6 or trans-11 18:1 under control or lactate-enriched (200 mM Na lactate) conditions for $24 \mathrm{~h}$. Of the 28 strains, Cutibacterium acnes (formerly Propionibacterium acnes) was the only bacterium found to produce trans-10 intermediates from 18:3n-3 and 18:2n-6, irrespective of the growth condition. To further assess the potential importance of this species in the trans-11 to trans-10 shift, different biomass ratios of Butyrivibrio fibrisolvens (as a trans-11 producer) and C. acnes were incubated in different growth media (control, low pH and 22:6n-3 enriched media) containing $40 \mu \mathrm{g} / \mathrm{mL}$ 18:2n-6. Under control conditions, a trans-10 shift, defined in the current study as trans-10/trans- $11 \geq 0.9$, occurred when the biomass of $C$. acnes represented between 90 and $98 \%$ of the inoculum. A low pH or addition of 22:6n-3 inhibited cis-9, trans-11 CLA and trans-10, cis-12 CLA formation by B. fibrisolvens and C. acnes, respectively, whereby $C$. acnes seemed to be more tolerant. This resulted in a decreased biomass of $C$. acnes required at inoculation to induce a trans-10 shift to 50\% (low pH) and 90\% (22:6n-3 addition).
\end{abstract}

Conclusions: Among the bacterial species studied, C. acnes was the only bacterium that have the metabolic ability to produce trans-10 intermediates from 18:3n-3 and 18:2n-6. Nevertheless, this experiment revealed that it is unlikely that $C$. acnes is the only or predominant species involved in the trans-11 to trans-10 shift in vivo.

Keywords: Biohydrogenation, Cuitbacterium acnes, Pure cultures, Rumen, trans-11 to trans-10 shift

\section{Background}

Ruminant diets commonly contain forages and concentrates, with mainly 18 -carbon unsaturated fatty acids (FA) (i.e. linolenic acid, 18:3n-3 and linoleic acid, 18:2n-6) [1]. Following ingestion, dietary lipids are hydrolyzed and the non-esterified FA are released into the rumen. Unsaturated FA are then converted to saturated FA by rumen bacteria

\footnotetext{
* Correspondence: Veerle.Fievez@UGent.be

†Lore Dewanckele and Jeyamalar Jeyanathan shared first authorship 'Laboratory for Animal Nutrition and Animal Product Quality (LANUPRO), Department of Animal Sciences and Aquatic Ecology, Ghent University, Ghent, Belgium

Full list of author information is available at the end of the article
}

via a process called biohydrogenation $[2,3]$. This involves several consecutive conversions via various pathways, resulting in the formation of a plethora of biohydrogenation intermediates, and ultimately in the formation of 18:0 [3]. The predominant biohydrogenation pathways of 18 : $3 n-3$ and 18:2n-6 involve the formation of intermediates containing a double bond in the trans configuration at the 11th carbon atom from the carboxyl end, further referred to as trans-11 intermediates (i.e. cis-9, trans-11, cis-15 conjugated linolenic acid (CLnA), trans-11, cis-15 18:2, cis-9, trans-11 conjugated linoleic acid (CLA) and trans-11 18:1) [3]. Nevertheless, under certain dietary conditions (i.e. high starch/low fiber diets or diets supplemented with marine

(c) The Author(s). 2020 Open Access This article is licensed under a Creative Commons Attribution 4.0 International License, which permits use, sharing, adaptation, distribution and reproduction in any medium or format, as long as you give appropriate credit to the original author(s) and the source, provide a link to the Creative Commons licence, and indicate if changes were made. The images or other third party material in this article are included in the article's Creative Commons licence, unless indicated otherwise in a credit line to the material. If material is not included in the article's Creative Commons licence and your intended use is not permitted by statutory regulation or exceeds the permitted use, you will need to obtain permission directly from the copyright holder. To view a copy of this licence, visit http://creativecommons.org/licenses/by/4.0/ The Creative Commons Public Domain Dedication waiver (http://creativecommons.org/publicdomain/zero/1.0/) applies to the data made available in this article, unless otherwise stated in a credit line to the data. 
oils or vegetable oils), a shift might occur in biohydrogenation pathway toward the formation of trans-10 intermediates (i.e. trans-10, cis-12, cis-15 CLnA, trans-10, cis-15 18: 2, trans-10, cis-12 CLA and trans-10 18:1) at the expense of trans-11 intermediates $[1,4,5]$, referred to as the trans11 to trans-10 shift.

The formation in the rumen of various biohydrogenation intermediates seems the responsibility of several bacterial species (e.g. [6, 7]). Although there might be other bacteria producing trans-11 intermediates, some bacteria involved in the trans-11 biohydrogenation pathway have been identified. Butyrivibrio fibrisolvens was the first ruminal bacterial species found to carry out biohydrogenation of 18:2n-6 and 18:3n-3 in vitro through the trans-11 pathway [6]. Nevertheless, further research identified other Butyrivibrio spp., Pseudobutyrivibrio spp. and Sharpea spp. as also capable of producing trans-11 intermediates (e.g. [8-10]). Several other bacterial species can also convert 18:2n-6 to cis-9, trans-11 CLA, e.g. species belonging to the genera Bifidobacterium [11], Lactobacillus [12], and Roseburia [13]. However, all members of the (Pseudo) butyrivibrio group, including $B$. fibrisolvens and $B$. proteoclasticus, and Sharpea spp. convert 18:2n-6 much more rapidly than other species $[10,14]$.

Trans-10 intermediates are particularly observed under specific dietary conditions in vivo (i.e. high starch/ low fiber diets and supplementation of marine oils or vegetable oils), which are often associated with milk fat depression [4, 15]. Despite the practical and economic relevance of the latter, the rumen bacteria responsible for the formation of trans-10 intermediates have not yet been unambiguously identified. Wallace et al. [16] suggested that Cutibactrium acnes (formerly Propionibacterium acnes [17]) is responsible for the formation of trans-10, cis-12 CLA from 18:2n-6. However, the relevance of this species in inducing a trans-10 shift in the rumen is questionable given its very low ruminal abundance $[14,18]$. Considering its increasing ruminal abundance in situations with milk fat depression [19-21], Megasphaera elsdenii was proposed as an alternative candidate responsible for ruminal trans-10, cis-12 CLA formation. However, conflicting results upon incubation with 18: $2 n-6[8,22]$ question its role in this process. Kemp et al. [23] observed formation of trans-10 18:1 from 18:2n-6 and $18: 3 n-3$ by Ruminococcus albus. Nevertheless, no other studies reported on the $18: 2 n-6$ and 18:3n-3 metabolism by R. albus since 1975 and therefore, its role in ruminal trans10 formation is unclear. Therefore, further research is needed to gain more information about the biohydrogenation ability and pathway of those bacteria.

Correlation analysis based on ruminal bacterial populations and milk [21, 24], blood [17] or rumen FA profiles $[17,25]$ revealed the possible contribution of several bacterial genera in ruminal formation of trans-10 intermediates, i.e. Acidaminococcus spp., Bifidobacterium spp., Carnobacterium spp., Dialister spp., and Lactobacillus spp. However, in vitro studies using pure cultures are needed to ascertain the capacity of those bacteria to produce trans-10 intermediates from 18:3n-3 or 18:2n-6. Next to this, as Streptococcus spp. and Selenomonas spp. often increase upon feeding high-grain diets [26, 27], which are often associated with a trans-11 to trans-10 shift [4]. Investigating the biohydrogenating ability of these bacteria could elucidate their potential involvement in ruminal trans-10 formation.

The majority of the bacteria potentially involved in ruminal trans-10 formation are related to ruminal lactate metabolism, which is often altered when feeding high-grain diets [28]. As such, lactate might influence the ruminal biohydrogenation process by affecting the metabolism of certain bacteria. Another potential route of trans-10 formation is via the formation of hydroxy FA. According to Devillard et al. [29], hydroxy FA produced by Roseburia spp. are converted to CLA by a mixed microbial community originating from human feces. As such, 18:3n-3 or 18:2n-6 might be converted to hydroxy FA by certain rumen species (e.g. S. bovis [8]), which might then be converted to trans-10 intermediates by other bacteria. Finally, trans-10 18:1 might, at least in part, originate from trans-11 18:1 in the rumen as Laverroux et al. [30] observed isomerization of trans11 18:1 to trans-10 18:1 by mixed cultures in vitro.

The aim of this study was to identify rumen bacteria that produce trans-10 intermediates from $18: 3 n-3$ or 18 : $2 n-6$, or their biohydrogenation and biohydrating intermediates. It was hypothesized that at least some of the investigated strains produce trans-10 intermediates. Additionally, the effect of supplementation of lactate to the medium on the metabolism of 18:2n-6 was investigated. It was hypothesized that lactate-utilizing bacteria would grow better under lactate-enriched conditions and would alter their metabolism and convert 18:2n-6 to trans-10 intermediates. Finally, the potential importance of trans-10 producing bacteria was investigated by using different biomass ratios of bacteria capable of producing cis-9, trans-11 CLA (B. fibrisolvens) and trans-10, cis-12 CLA (C. acnes) from 18:2n-6 under different in vitro conditions.

\section{Results}

Metabolism of linolenic, linoleic, and vaccenic acid by individual species of rumen bacteria (Exp. 1)

The metabolism of 18:3n-3, 18:2n-6 and trans-11 18:1 by the 28 individual strains is presented in Table 1 . Nine strains metabolized 18:3n-3 and 18:2n-6 during a $24-\mathrm{h}$ incubation period. Butyrivibrio fibrisolvens D1 showed an accumulation of trans-11 intermediates (i.e. cis-9, trans-11, cis-15 CLnA, trans-11, cis-15 18:2 and trans-11 $18: 1)$, when incubated with $18: 3 n-3$ or $18: 2 n-6$. When 
Table 1 Metabolism of $18: 3 n-3$ and $18: 2 n-6^{\text {a }}$ by different bacterial strains during $24 \mathrm{~h}$ of incubation under control growth conditions (Exp. 1). Blank cells related to 18:3n-3 and 18:2n-6 indicate no metabolism takes place by these strains

\begin{tabular}{|c|c|c|c|c|c|c|}
\hline \multirow[t]{2}{*}{ Strain } & \multirow{2}{*}{$\begin{array}{l}\text { Total VFA formed } \\
(\mu \mathrm{mol} / \text { tube; } \\
\text { mean } \pm S D)^{b}\end{array}$} & \multirow{2}{*}{$\begin{array}{l}\text { VFA } \\
\text { products }\end{array}$} & \multicolumn{2}{|l|}{$18: 3 n-3$} & \multicolumn{2}{|l|}{$18: 2 n-6$} \\
\hline & & & $\begin{array}{l}\% \text { metabolized } \\
(\text { mean } \pm \mathrm{SD})^{\mathrm{d}}\end{array}$ & $\begin{array}{l}\text { Products formed } \\
(\% \text { of total } \\
\text { intermediates } \\
\text { formed and } \\
\text { remaining } 18: 3 n-3)^{\mathrm{e}, \mathrm{f}}\end{array}$ & $\begin{array}{l}\% \text { metabolized } \\
(\text { mean } \pm S D)^{d}\end{array}$ & $\begin{array}{l}\text { Products formed } \\
\text { (\% of total } \\
\text { intermediates and } \\
\text { remaining } \\
18: 2 n-6)^{\mathrm{e}, \mathrm{f}}\end{array}$ \\
\hline $\begin{array}{l}\text { Acidaminococcus } \\
\text { fermentans VR4 }\end{array}$ & $54 \pm 9.5$ & $A, B$ & & & & \\
\hline $\begin{array}{l}\text { Acidaminococcus } \\
\text { intestini ADV } 255.99\end{array}$ & $55 \pm 13.0$ & $A, B$ & & & & \\
\hline $\begin{array}{l}\text { Bifidobacterium } \\
\text { adolescentis RU } 424\end{array}$ & $251 \pm 5.4$ & A & $79.8 \pm 13.94$ & $10-\mathrm{OH} \Delta 12,15-18: 2(45 \%)$ & $76.8 \pm 6.86$ & $10-\mathrm{OH} \triangle 12-18: 1(62 \%)$ \\
\hline $\begin{array}{l}\text { Bifidobacterium } \\
\text { pseudolongum RU224 }\end{array}$ & $210 \pm 18.5$ & A & $26.7 \pm 14.27$ & $10-\mathrm{OH} \Delta 12,15-18: 2(13 \%)$ & $23.1 \pm 8.56$ & $10-\mathrm{OH} \triangle 12-18: 1(15 \%)$ \\
\hline \multirow{3}{*}{$\begin{array}{l}\text { Butyrivibrio } \\
\text { fibrisolvens D1 }\end{array}$} & \multirow[t]{3}{*}{$102 \pm 45.9$} & \multirow[t]{3}{*}{$B, A$} & \multirow[t]{3}{*}{$99.3 \pm 0.43$} & $c 9, t 11, c 15$ CLnA (48\%) & \multirow[t]{3}{*}{$98.0 \pm 0.40$} & \multirow[t]{3}{*}{$t 11$ 18:1 (89\%) } \\
\hline & & & & $t 11, c 1518: 2(43 \%)$ & & \\
\hline & & & & t11 C18:1 (6\%) & & \\
\hline \multirow{5}{*}{$\begin{array}{l}\text { Butyrivibrio } \\
\text { proteoclasticus P18 }\end{array}$} & \multirow[t]{5}{*}{$169 \pm 12.7$} & \multirow[t]{5}{*}{$B, A$} & \multirow[t]{5}{*}{$98.9 \pm 0.03$} & $c 9 / t 13 / t 14$ 18:19 (42\%) & \multirow[t]{5}{*}{$98.4 \pm 0.48$} & 18:0 (76\%) \\
\hline & & & & $t 15 / c 11$ 18:19 (21\%) & & $t 11$ 18:1 (17\%) \\
\hline & & & & C15 18:1 (17\%) & & \\
\hline & & & & $18: 0(6 \%)$ & & \\
\hline & & & & $\mathrm{t} 11, \mathrm{c} 15$ 18:2 (6\%) & & \\
\hline Lactobacillus ruminis RF1 & $30 \pm 17.2$ & $A, P$ & & & & \\
\hline Lactobacillus ruminis RF2 & $14 \pm 11.9$ & $A, P$ & & & & \\
\hline \multirow{2}{*}{$\begin{array}{l}\text { Cutibacterium acnes } \\
\text { DSM } 1897\end{array}$} & \multirow[t]{2}{*}{$99 \pm 43.7$} & \multirow[t]{2}{*}{$P, A$} & \multirow[t]{2}{*}{$86.5 \pm 15.83$} & $\Delta 11,13,15-18: 3(50 \%)$ & \multirow[t]{2}{*}{$88.4 \pm 7.16$} & $t 10, c 12$ CLA (75\%) \\
\hline & & & & $t 10, c 12, c 15$ CLnA (5\%) & & 10-OH 12-18:1 (7\%) \\
\hline Ruminococcus albus 7 & $24 \pm 8.6$ & A & & & & \\
\hline $\begin{array}{l}\text { Streptococcus equinus } \\
\text { Pearl } 11\end{array}$ & $18 \pm 5.2$ & A & $21.6 \pm 10.58$ & 13-OH $\triangle 9,15-18: 2(6 \%)$ & $84.0 \pm 2.95$ & 13-OH $\Delta 9-18: 1$ (69\%) \\
\hline \multirow{2}{*}{$\begin{array}{l}\text { Streptococcus gallolyticus } \\
\text { DSM } 16831\end{array}$} & \multirow[t]{2}{*}{$16 \pm 5.1$} & \multirow[t]{2}{*}{ A } & \multirow[t]{2}{*}{$96.3 \pm 1.49$} & \multirow[t]{2}{*}{ 13-OH $\Delta 9,15-18: 2(86 \%)$} & \multirow[t]{2}{*}{$89.8 \pm 2.99$} & 13-OH $\triangle 9-18: 1$ (47\%) \\
\hline & & & & & & $\Delta 9,14-18: 2(32 \%)$ \\
\hline Megasphaera elsdenii B159 & $147 \pm 5.6$ & B & & & & \\
\hline Megasphaera elsdenii T81 & $126 \pm 6.1$ & B & & & & \\
\hline Megasphaera elsdenii LC1 & $121 \pm 11.2$ & $B, A$ & & & & \\
\hline Megasphaera elsdenii 2602A & $191 \pm 21.2$ & $B, P$ & $32.7 \pm 8.92$ & 13-OH $\triangle 9,15-18: 2$ (19\%) & $81.8 \pm 3.71$ & 13-OH $\triangle 9-18: 1$ (63\%) \\
\hline & & & & & & $\Delta 9,14-18: 2(5 \%)$ \\
\hline Megasphaera elsdenii 3016B & $138 \pm 10.5$ & B & & & & \\
\hline Megasphaera elsdenii 3218A & $134 \pm 7.9$ & B & & & & \\
\hline Megasphaera elsdenii 3436A & $117 \pm 4.3$ & $\mathrm{~B}$ & & & & \\
\hline Megasphaera elsdenii 4251 & $125 \pm 6.8$ & B & & & & \\
\hline Megasphaera elsdenii 4257 & $124 \pm 5.3$ & B & & & & \\
\hline Megasphaera elsdenii 4296 & $120 \pm 5.5$ & $\mathrm{~B}$ & & & & \\
\hline Megasphaera elsdenii 4400 & $58 \pm 25.8$ & $A, P$ & & & & \\
\hline Megasphaera elsdenii 5045 & $128 \pm 6.6$ & B & & & & \\
\hline Megasphaera elsdenii 5052B & $63 \pm 9.9$ & A & $60.9 \pm 11.21$ & $10-\mathrm{OH} \Delta 12,15-18: 2(34 \%)$ & $88.0 \pm 2.20$ & 13-OH $\triangle 9-18: 1$ (42\%) \\
\hline & & & & & & 10-OH $\triangle 12-18: 1$ (24\%) \\
\hline
\end{tabular}


Table 1 Metabolism of 18:3n-3 and 18:2n- $6^{\mathrm{a}}$ by different bacterial strains during $24 \mathrm{~h}$ of incubation under control growth conditions (Exp. 1). Blank cells related to 18:3n-3 and 18:2n-6 indicate no metabolism takes place by these strains (Continued)

\begin{tabular}{|c|c|c|c|c|c|c|}
\hline \multirow[t]{2}{*}{ Strain } & \multirow{2}{*}{$\begin{array}{l}\text { Total VFA formed } \\
(\mu \mathrm{mol} / \mathrm{tube} \\
\text { mean } \pm S D)^{b}\end{array}$} & \multirow{2}{*}{$\begin{array}{l}\text { VFA } \\
\text { products }\end{array}$} & \multicolumn{2}{|l|}{$18: 3 n-3$} & \multicolumn{2}{|l|}{$18: 2 n-6$} \\
\hline & & & $\begin{array}{l}\% \text { metabolized } \\
(\text { mean } \pm \text { SD })^{d}\end{array}$ & $\begin{array}{l}\text { Products formed } \\
(\% \text { of total } \\
\text { intermediates } \\
\text { formed and } \\
\text { remaining } 18: 3 n-3)^{e, f}\end{array}$ & $\begin{array}{l}\% \text { metabolized } \\
(\text { mean } \pm S D)^{d}\end{array}$ & $\begin{array}{l}\text { Products formed } \\
\text { (\% of total } \\
\text { intermediates and } \\
\text { remaining } \\
18: 2 n-6)^{\mathrm{e}, \mathrm{f}}\end{array}$ \\
\hline $\begin{array}{l}\text { Selenomonas ruminantium } \\
\text { GA-192 }\end{array}$ & $83 \pm 12.0$ & \multicolumn{5}{|l|}{$P, A$} \\
\hline Selenomonas ruminantium PC 18 & $241 \pm 31.8$ & \multicolumn{5}{|l|}{$P, A$} \\
\hline \multicolumn{7}{|c|}{ 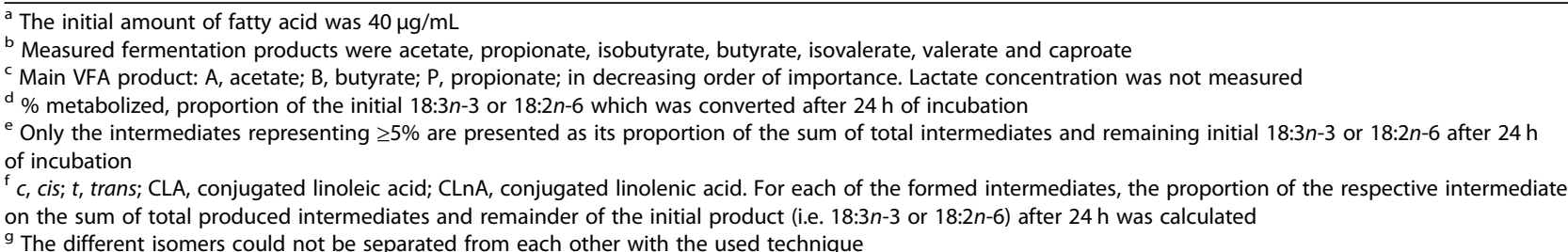 } \\
\hline
\end{tabular}

incubated with 18:2n-6, B. proteoclasticus P18 additionally produced 18:0 besides trans-11 18:1. In contrast to $B$. fibrisolvens, there was only $6 \%$ of trans-11 (trans-11, cis15 18:2) intermediates after $24 \mathrm{~h}$ of incubation of $B$. proteoclasticus with 18:3n-3. Interestingly, C. acnes DSM 1897 was the only strain found to produce trans-10, cis12, cis-15 CLnA and trans-10, cis-12 CLA from 18:3n-3 and 18:2n-6, respectively. However, trans-10, cis- 12 , cis- 15 CLnA represented only $5 \%$ of the products formed from 18:3n-3. When incubated with $18: 3 n-3, C$. acnes additionally produced $\Delta 11,13,15-18: 3$, which represented $50 \%$ of the products formed. The final products of Bifidobacterium adolescentis RU 424, Bifidobacterium pseudolongum RU224, Streptococcus gallolyticus DSM 16831, Streptococcus equinus Pearl 11, and M. elsdenii 2602A and 5052B were mainly hydroxy FA, with the hydroxyl group located at the 10th (i.e. B. adolescentis, B. pseudolongum and $M$. elsdenii 5052B) and/or at the 13th (i.e. S. equinus, $S$. gallolyticus, and M. elsdenii 2602A and 5052B) carbon atom from the carboxyl end. Trans-11 18:1 was only converted by B. proteoclasticus P18 to $18: 0$. After $24 \mathrm{~h}$ of incubation, $51.0 \pm 9.52 \%($ mean $\pm \mathrm{SD})$ of the initial trans11 18:1 was metabolized to 18:0. No other bacteria metabolized trans-11 18:1 (data not shown).

\section{Influence of lactate on metabolism of linoleic acid by individual species of rumen bacteria (Exp. 2)}

Supplementation of lactate to the growth medium only affected the metabolism of 18:2n-6 by two of the studied strains, i.e. M. elsdenii 5052B and B. pseudolongum RU224 (Table 2). With M. elsdenii 5052B, supplementation of lactate to the medium decreased both the OD value $(P=0.013$; Table $S 1)$ as well as the metabolized proportion of $18: 2 n-6$ after $24 \mathrm{~h}$ of incubation (from 88.01 to $74.35 \% ; P=0.006$, Table 2). With B. pseudolongum RU224, supplementation of lactate increased the metabolized proportion of $18: 2 n-6$ after $24 \mathrm{~h}$ of incubation (from 23.06 to $38.41 \% ; P=0.033$, Table 2). However, with both bacterial strains, the accumulated intermediates remained the same as under control growth conditions. Although differences were also observed for several other strains in OD value (Table S1) and total VFA produced (Table S2) after $24 \mathrm{~h}$ of incubation between the control treatment and the lactate-enriched medium, particularly for the lactate-utilizing bacteria M. elsdenii and Selenomonas ruminantium, no differences in metabolized proportion of $18: 2 n-6$ or in accumulated intermediates were observed for any of the other strains (Table 2).

\section{Effect of growth medium on metabolism of $18: 2 n-6$ by monocultures of B. fibrisolvens D1 and C. acnes DSM 1897 (Exp. 3)}

The growth medium generally affected the metabolism of 18:2n-6 during the $24 \mathrm{~h}$ incubation period, the mean proportions of 18:2n-6, cis-9, trans-11 CLA, trans-11 18:1, and trans-10, cis-12 CLA differed between bacterial species $(P$-values for interaction effect ranging from $<0.001$ to 0.011; Table 3). With B. fibrisolvens, $18: 2 n-6$ was almost completely metabolized after $8 \mathrm{~h}$ of incubation under control growth conditions (Fig. 1a). This disappearance of 18: $2 n-6$ was accompanied by a transient accumulation of cis9, trans-11 CLA (Fig. 1b), which was further transformed to trans-11 18:1 (Fig. 1c). No trans-10, cis-12 CLA (Fig. 1d) or 18:0 (data not shown) was formed by $B$. fibrisolvens. The average 18:2n-6 proportion was higher $(P<0.001)$ with the low $\mathrm{pH}$ and DHA-enriched media compared with the control medium (Table 3, Fig. 1a), which implies inhibition of the formation of cis-9, trans-11 CLA (Fig. 1b). These conditions also inhibited further transformation of cis-9, trans-11 CLA to trans-11 18:1, as the proportion of trans-11 18:1 was lower $(P<0.001)$ with the low $\mathrm{pH}$ and 
Table 2 Influence of lactate on metabolism of $18: 2 n-6^{a}$ by different bacterial strains during $24 \mathrm{~h}$ of incubation (Exp. 2)

\begin{tabular}{|c|c|c|c|c|c|c|}
\hline \multirow[t]{2}{*}{ Strain $^{b}$} & \multicolumn{2}{|l|}{ Control } & \multicolumn{2}{|l|}{ Lactate } & \multirow[t]{2}{*}{ SEM } & \multirow[t]{2}{*}{$P$-value } \\
\hline & $\%$ metabolized $^{c}$ & $\begin{array}{l}\text { Main products formed } \\
\text { (\% of total intermediates } \\
\text { and remaining } 18: 2 n-6)^{\text {d,e }}\end{array}$ & $\%$ metabolized $^{c}$ & $\begin{array}{l}\text { Main products formed } \\
\text { (\% of total intermediates } \\
\text { and remaining } 18: 2 n-6)^{d, e}\end{array}$ & & \\
\hline Bifidobacterium adolescentis RU 424 & 76.75 & $10-\mathrm{OH} \triangle 12-18: 1(62 \%)$ & 82.22 & $10-\mathrm{OH} \triangle 12-18: 1(67 \%)$ & 2.859 & 0.234 \\
\hline Bifidobacterium pseudolongum RU224 & 23.06 & 10-OH $\triangle 12-18: 1$ (15\%) & 38.41 & 10-OH $\triangle 12-18: 1(18 \%)$ & 3.716 & 0.033 \\
\hline Butyrivibrio fibrisolvens D1 & 98.00 & $t 11$ 18:1 (89\%) & 97.90 & $t 11$ 18:1 (90\%) & 0.217 & 0.765 \\
\hline \multirow[t]{2}{*}{ Butyrivibrio proteoclasticus P18 } & 98.35 & 18:0 (69\%) & 98.54 & 18:0 (76\%) & 0.248 & 0.295 \\
\hline & & $t 11$ 18:1 (22\%) & & $t 11$ 18:1 (17\%) & & \\
\hline \multirow[t]{2}{*}{ Cutibacterium acnes DSM 1897} & 88.41 & $\boldsymbol{t} \mathbf{1 0}, \mathrm{C} 12 \mathrm{CLA}(75 \%)$ & 60.79 & $\boldsymbol{t} \mathbf{1 0}, \mathrm{C} 12 \mathrm{CLA}(39 \%)$ & 20.522 & 0.395 \\
\hline & & 10-OH 12-18:1 (7\%) & & 10-OH 12-18:1 (5\%) & & \\
\hline Streptococcus equinus Pearl 11 & 83.96 & 13-OH $\Delta 9-18: 1$ (69\%) & 80.92 & 13-OH $\triangle 9-18: 1$ (64\%) & 1.493 & 0.223 \\
\hline \multirow[t]{2}{*}{ Streptococcus gallolyticus DSM 16831} & 89.75 & 13-OH $\triangle 9-18: 1$ (47\%) & 92.77 & $\Delta 9,14-18: 2(56 \%)$ & 1.877 & 0.319 \\
\hline & & $\Delta 9,14-18: 2(32 \%)$ & & 13-OH $\triangle 9-18: 1$ (27\%) & & \\
\hline \multirow[t]{3}{*}{ Megasphaera elsdenii 2602A } & 81.82 & 13-OH $\triangle 9-18: 1$ (63\%) & 79.13 & 13-OH $\Delta 9-18: 1$ (50\%) & 2.287 & 0.452 \\
\hline & & $\Delta 9,14-18: 2(5 \%)$ & & $\Delta 9,14-18: 2(10 \%)$ & & \\
\hline & & & & c9, t13, t14 18:1 (7\%) & & \\
\hline \multirow[t]{3}{*}{ Megasphaera elsdenii 5052B } & 88.01 & 13-OH $\triangle 9-18: 1$ (42\%) & 74.35 & 13-OH $\Delta 9-18: 1$ (34\%) & 1.786 & 0.006 \\
\hline & & 10-OH $\triangle 12-18: 1$ (24\%) & & 10-OH $\triangle 12-18: 1$ (16\%) & & \\
\hline & & & & c9, t13, t14 18:1 (5\%) & & \\
\hline
\end{tabular}

a The initial amount of $18: 2 n-6$ was $40 \mu \mathrm{g} / \mathrm{mL}$

${ }^{b}$ Only the strains which metabolized $18: 2 n-6$ are shown

c $\%$ metabolized, proportion of the initial 18:2n-6 which was converted after $24 \mathrm{~h}$ of incubation

${ }^{d}$ Only the intermediates representing $\geq 5 \%$ of the sum of total intermediates and remaining initial $18: 2 n-6$ after $24 \mathrm{~h}$ of incubation are presented in this table

${ }^{\text {e }} c$, cis; $t$, trans; CLA, conjugated linoleic acid. For each of the formed intermediates, the proportion of the respective intermediate on the sum of total produced intermediates and remainder of 18:2n-6 after $24 \mathrm{~h}$ was calculated

DHA-enriched media compared with the control medium (Table 3, Fig. 1c).

Under control growth conditions, $C$. acnes converted 18:2n-6 to trans-10, cis-12 CLA, but this conversion to CLA was more slowly compared to $B$. fibrisolvens (Table 3, Fig. 1a and e). A low pH or addition of DHA to the medium increased the mean proportion of residual 18:2n-6 $(P<0.001$; Table 3 , Fig. 1 e), which was accompanied with a decreased proportion of trans-10, cis-12 CLA $(P<0.001$; Table 3, Fig. 1h). This implies inhibition of the formation of trans-10, cis-12 CLA by low $\mathrm{pH}$ and by DHA. This inhibition of 18:2n-6 isomerization was less pronounced with $C$. acnes compared with $B$. fibrisolvens. There was no formation of cis-9, trans-11 CLA (Fig. 1f), trans-11 18:1 (Fig. 1g), trans-10 18:1 (data not shown), or 18:0 (data not shown) by C. acnes.

Table 3 Average proportions ${ }^{\mathrm{a}}$ (\% of total intermediates and 18:2n-6) of 18:2n-6 and its biohydrogenation intermediates over a $24 \mathrm{~h}$ incubation period under different growth conditions ${ }^{\mathrm{b}}$ with mono-cultures of Butyrivibrio fibrisolvens D1 or Propionibacterium acnes DSM 1897 (Exp. 3)

\begin{tabular}{|c|c|c|c|c|c|c|c|c|c|c|}
\hline & \multicolumn{3}{|c|}{ B. fibrisolvens } & \multicolumn{3}{|l|}{ C. acnes } & \multirow[t]{2}{*}{ SEM } & \multicolumn{3}{|l|}{$P$-value } \\
\hline & Control & Low pH & $\mathrm{DHA}$ & Control & Low pH & $\mathrm{DHA}$ & & Bacterium & $\begin{array}{l}\text { Growth } \\
\text { medium }\end{array}$ & $\begin{array}{l}\text { Bacterium } \times \\
\text { growth medium }\end{array}$ \\
\hline $18: 2 n-6$ & $20.72^{\beta *}$ & $73.86^{a *}$ & $71.49^{a *}$ & $77.00^{\beta}$ & $86.88^{a}$ & $95.21^{a}$ & 3.918 & $<0.001$ & $<0.001$ & $<0.001$ \\
\hline cis-9, trans-11 CLA ${ }^{c}$ & $8.01^{\beta}$ & $25.49^{a *}$ & $27.24^{a *}$ & 0.54 & 0.41 & 0.54 & 3.273 & $<0.001$ & 0.013 & 0.011 \\
\hline trans-11 18:1 & $71.10^{a *}$ & $0.55^{\beta}$ & $1.08^{\beta}$ & 0.64 & 4.46 & 0.33 & 1.959 & $<0.001$ & $<0.001$ & $<0.001$ \\
\hline trans-10, cis-12 CLA & $<0.01^{*}$ & $<0.01^{*}$ & 0.40 & $21.81^{a}$ & $8.25^{\beta}$ & $3.76^{\beta}$ & 2.073 & $<0.001$ & 0.001 & $<0.001$ \\
\hline
\end{tabular}

a The average proportions of FA over the $24 \mathrm{~h}$ incubation period were computed as the area under the curve divided by the total duration of incubation ( $24 \mathrm{~h}$ ), using the individual measured proportions for each FA at the different sampling times

${ }^{\mathrm{b}}$ Low $\mathrm{pH}$, control medium with $\mathrm{pH}$ adjusted to 5.5; DHA (docosahexaenoic acid), control medium containing $40 \mu \mathrm{g} / \mathrm{mL}$ of $22: 6 n-3$; all growth media contained $40 \mu \mathrm{g} / \mathrm{mL}$ of $18: 2 n-6$

c CLA, conjugated linoleic acid

a, $\beta$ Means differ $(P<0.05)$ between growth media within the same bacterial species

* Means differ $(P<0.05)$ between $B$. fibrisolvens and $C$. acnes within the same growth medium 
Butyrivibrio fibrisolvens
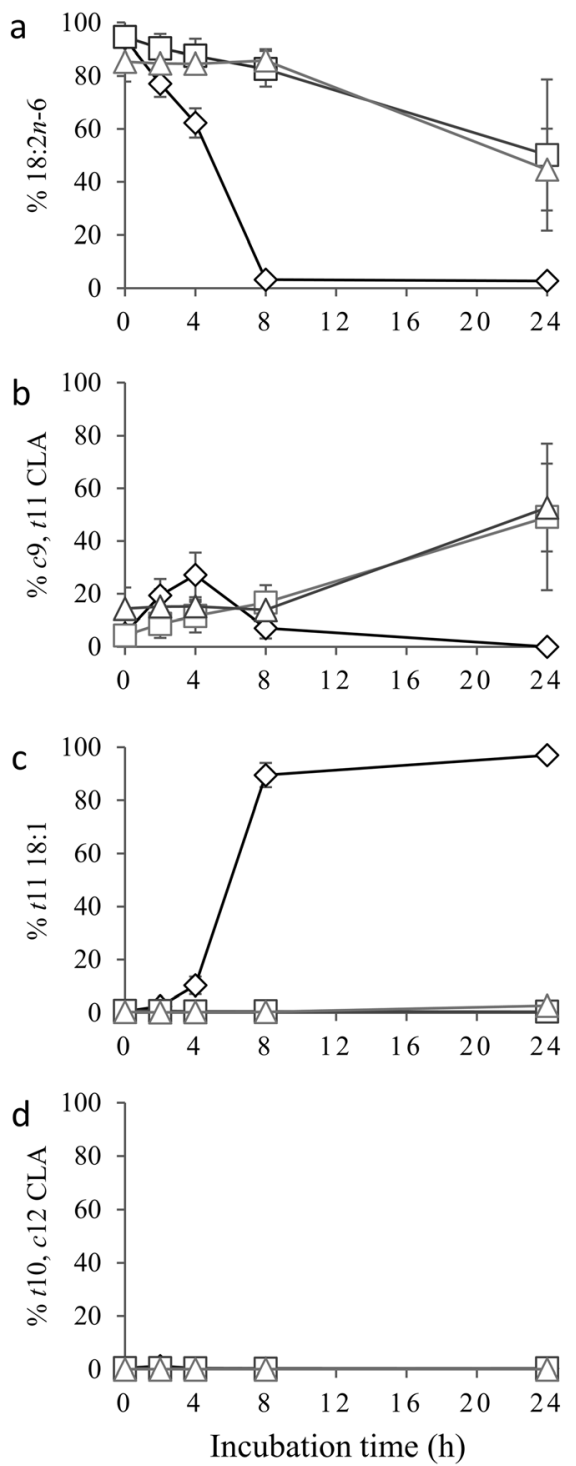

Propionibacterium acnes
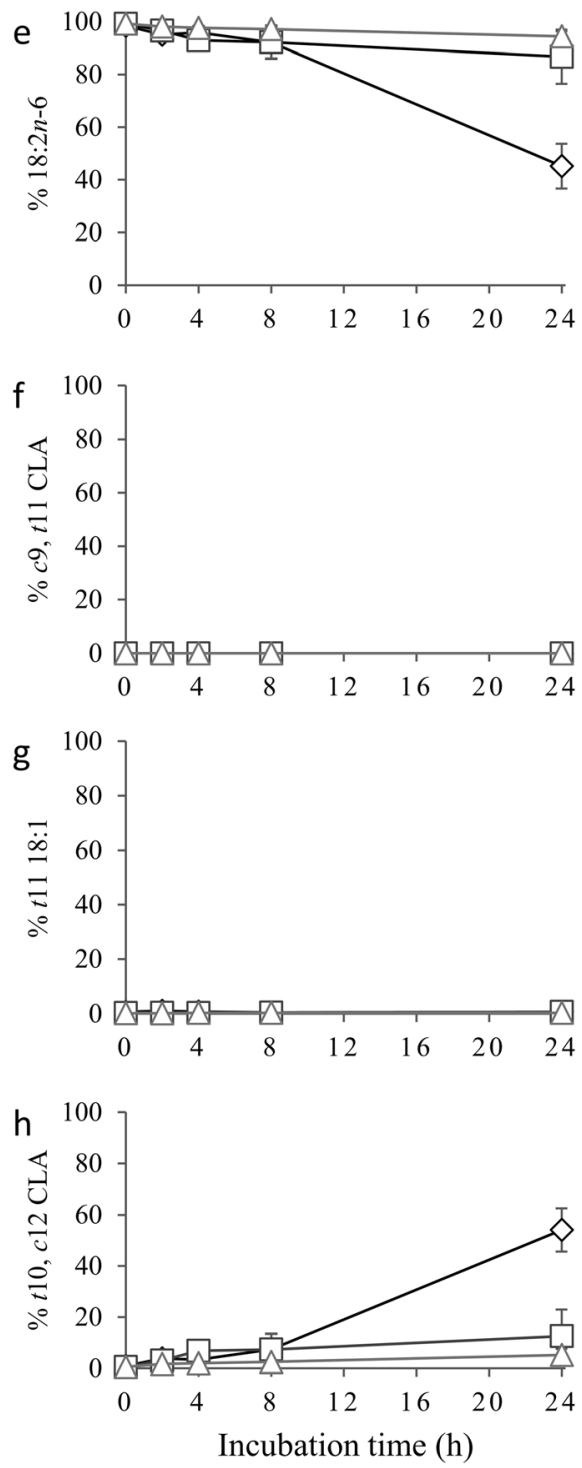

Fig. 1 Effect of growth medium on the proportion of 18:2n-6 and its biohydrogenation intermediates (\% of total intermediates and 18:2n-6) during a $24 \mathrm{~h}$ incubation period with Butyrivibrio fibrisolvens D1 (left; a-d) and Cutibacterium acnes DSM 1897 (right; e-h) (Exp. 3). The initial concentration of 18:2n-6 was $40 \mu \mathrm{g} / \mathrm{mL}$. Control medium (diamond); low pH medium (square), control medium with pH adjusted to 5.5; DHAenriched (docosahexaenoic acid) medium (triangle), control medium containing $40 \mu \mathrm{g} / \mathrm{mL}$ of 22:6n-3. c, cis; $t$, trans; CLA, conjugated linoleic acid

Effect of ratio of B. fibrisolvens D1 to C. acnes DSM 1897 in the inoculum in combination with varying growth media on the trans-11 to trans-10 shift and volatile fatty acid production (Exp. 4)

The biomass ratio of $B$. fibrisolvens to $C$. acnes in the inoculum had an effect on the disappearance of $18: 2 n-6$ (Table $4 ; P<0.05$ ). The mean proportion of $18: 2 n-6$ over the $24 \mathrm{~h}$ incubation period increased with increasing biomass amounts of $C$. acnes in the inoculum $(P<0.001)$. Increasing biomass of $C$. acnes also increased the accumulation of trans-10, cis-12 CLA $(P \leq 0.007)$ at the expense of cis-9, trans-11 CLA $(P=0.013)$, with a trans-11 to trans-
10 shift, defined in the current experiment as trans-10/ trans-11 $\geq 0.9$, occurring when the relative biomass of $C$. acnes in the inoculum was between 90 and 98\% (Table 4, Fig. 2a). In mono-cultures of C. acnes (Exp. 3 and Exp. 4), metabolism of 18:2n-6 resulted in the accumulation of trans-10, cis-12 CLA, whereas in co-culture with B. fibrisolvens (Exp. 4), trans-10, cis-12 CLA was partially further metabolized to trans-10 18:1 (Table 4), indicating that the latter bacterium is responsible for the conversion of trans10, cis-12 CLA to trans-10 18:1.

The effect of the ratio of $B$. fibrisolvens to $C$. acnes in the inoculum on $18: 2 n-6$ metabolism depended on the 
Table 4 Average proportions ${ }^{\mathrm{a}}$ (\% of total intermediates and 18:2n-6) of 18:2n- $6^{\mathrm{b}}$ and its biohydrogenation intermediates over a $24 \mathrm{~h}$ incubation period with different ratios of Butyrivibrio fibrisolvens D1 to Propionibacterium acnes DSM 1897 in the inoculum under control growth conditions (Exp. 4)

\begin{tabular}{|c|c|c|c|c|c|c|c|c|c|}
\hline & \multicolumn{6}{|c|}{ B. fibrisolvens (\%)/C. acnes (\%) } & \multirow[t]{2}{*}{ SEM } & \multicolumn{2}{|l|}{$P$-value } \\
\hline & $100 / 0$ & $50 / 50$ & $10 / 90$ & $2 / 98$ & $0.4 / 99.6$ & $0 / 100$ & & Linear & Quadratic \\
\hline $18: 2 n-6$ & 12.35 & 19.59 & 34.90 & 49.57 & 53.30 & 66.65 & 12.928 & $<0.001$ & $<0.001$ \\
\hline cis-9, trans-11 CLA & 19.22 & 22.44 & 10.57 & 8.89 & 4.15 & 0.02 & 7.797 & 0.013 & 0.090 \\
\hline trans-11 18:1 & 67.98 & 51.72 & 34.59 & 14.22 & 15.62 & 0.41 & 14.947 & $<0.001$ & 0.035 \\
\hline trans-10, cis-12 CLA ${ }^{c}$ & $<0.01$ & 3.03 & 11.55 & 20.08 & 19.13 & 32.92 & 3.123 & $<0.001$ & 0.007 \\
\hline trans-10 18:1 & 0.45 & 3.22 & 8.39 & 7.24 & 7.80 & $<0.01$ & 4.090 & 0.014 & 0.471 \\
\hline trans-10/trans $-11^{d}$ & - & 0.08 & 0.48 & 3.00 & 4.04 & - & 4.436 & $<0.001$ & $<0.001$ \\
\hline
\end{tabular}

${ }^{a}$ The average proportions of FA over the $24 \mathrm{~h}$ incubation period were computed as the area under the curve divided by the total duration of incubation ( $24 \mathrm{~h}$ ), using the individual measured proportions for each FA at the different sampling times

${ }^{b}$ The initial amount of $18: 2 n-6$ was $40 \mu \mathrm{g} / \mathrm{mL}$

c CLA, conjugated linoleic acid

${ }^{d}$ Ratio of trans-10 intermediates (trans-10, cis-12 CLA + trans-10 18:1) to trans-11 intermediates (cis-9, trans-11 CLA + trans-11 18:1)

$-:$ The ratio is not relevant for mono-cultures as trans-11 and trans-10 are produced exclusively with mono-cultures of $B$. fibrisolvens and $C$. acnes, respectively

growth medium (Table 5; $P<0.05$ ). With the low $\mathrm{pH}$ medium or the DHA-enriched medium, an increase in residual 18:2n-6 after $24 \mathrm{~h}$ of incubation was observed compared to the control medium, which implies that a low $\mathrm{pH}$ or the addition of DHA to the medium reduced the rate of 18:2n-6 disappearance (Table 5). With the DHA-enriched medium, the amount of 18:2n-6 after the $24 \mathrm{~h}$ incubation period increased with increasing biomass of $C$. acnes in the inoculum $(P \leq 0.014)$, which was also the case under control conditions. In contrast to this, with the low $\mathrm{pH}$ medium, the amount of $18: 2 n-6$ after $24 \mathrm{~h}$ of incubation was relatively constant, irrespective of the bacterial ratio $(P \geq 0.05)$.

Under control conditions, a trans-11 to trans-10 shift (i.e. trans-10/trans- $11 \geq 0.9$ ) was observed when $C$. acnes represented between 90 and $98 \%$ of the inoculum biomass. With the addition of DHA, this shift already occurred at lower biomass proportions of $C$. acnes (i.e. between 50 and 90\%; Table 5, Fig. 2c vs. Figure 2a). The biomass proportion of $C$. acnes necessary to induce this shift was further decreased with the low $\mathrm{pH}$ medium, in which a relative biomass proportion of $50 \%$ C. acnes in the inoculum was sufficient to induce a trans-11 to trans-10 shift (Table 5, Fig. 2b vs. Figure 2a).

The mono-culture experiment (Exp. 3) revealed that B. fibrisolvens primarily produced butyric acid and $C$. acnes primarily generated propionic acid (data not shown). Under control growth conditions in experiment 4 , there was a linear increase in propionic acid production $(P<0.001)$ and a linear decrease in butyric acid production $(P=0.022)$ with increasing relative biomass of $C$. acnes in the inoculum (Table S3). Figure 3 shows that there was a delay in butyric acid production under control growth conditions in the first $8 \mathrm{~h}$ when the relative biomass of $C$. acnes in the inoculum was $90 \%$ or higher (Fig. 3c-e). A low pH or the addition of DHA to the medium reduced the net production of propionic acid and butyric acid by $C$. acnes and B. fibrisolvens, respectively $(P<0.05$; Table S3). The inhibitory effect of low $\mathrm{pH}$ on VFA production was higher compared to the addition of DHA. Moreover, the reduction in VFA production due to low $\mathrm{pH}$ and DHA addition was more pronounced for butyric acid as compared to propionic acid.

\section{Metabolism of hydration intermediates by mixed rumen inoculum (Exp. 5)}

The hydroxy FA formed by B. adolescentis RU 424, B. pseudolongum RU224, S. gallolyticus DSM 16831, S. equinus Pearl 11, and M. elsdenii 2602A and 5052B were extensively converted by the mixed rumen community during $24 \mathrm{~h}$ of incubation, although in each incubation flask, hydroxy FA remained at the end of the incubation $(<6 \%$, data not shown). Products formed were 18:0 and several unknown FA with a retention time close to that of the initial hydroxy FA. Nevertheless, the mixed rumen inoculum did not produce any trans-10 intermediates from the hydroxy FA during $24 \mathrm{~h}$ of incubation.

\section{Discussion}

Metabolism of linolenic, linoleic and vaccenic acid by pure cultures of individual rumen bacteria

In the rumen, $18: 3 n-3$ and $18: 2 n-6$ are converted to $18: 0$ via a process called biohydrogenation [3]. Their most common biohydrogenation pathway is via the formation of trans-11 intermediates. However, under certain dietary conditions, a shift in biohydrogenation pathway occurs toward the formation of trans-10 intermediates at the expense of trans-11 intermediates, which is often associated with milk fat depression $[4,5]$. Despite the practical and economic relevance of the latter, the rumen bacteria responsible for the formation of trans-10 


\section{a Control medium}

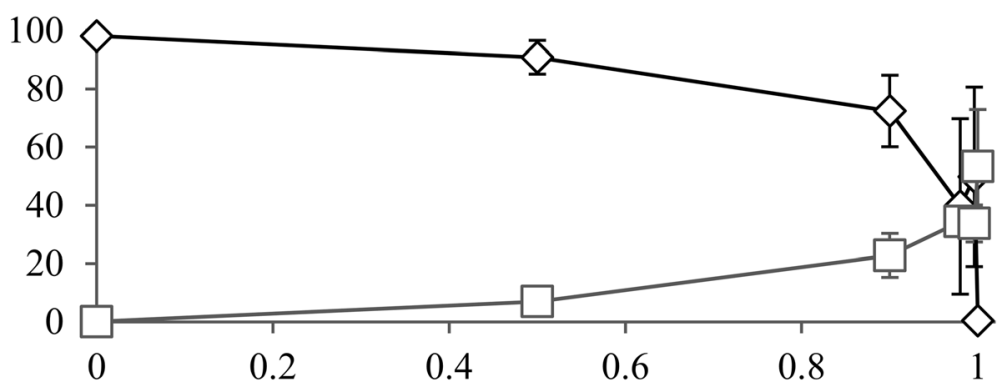

b Low pH medium

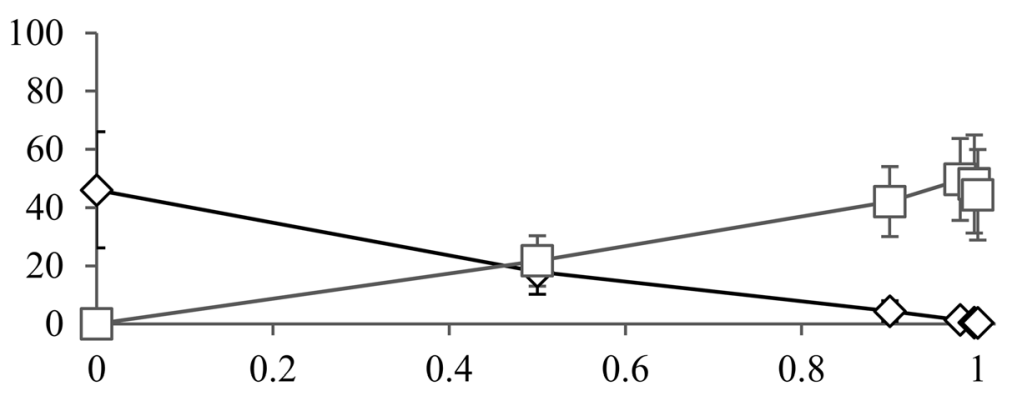

c DHA medium

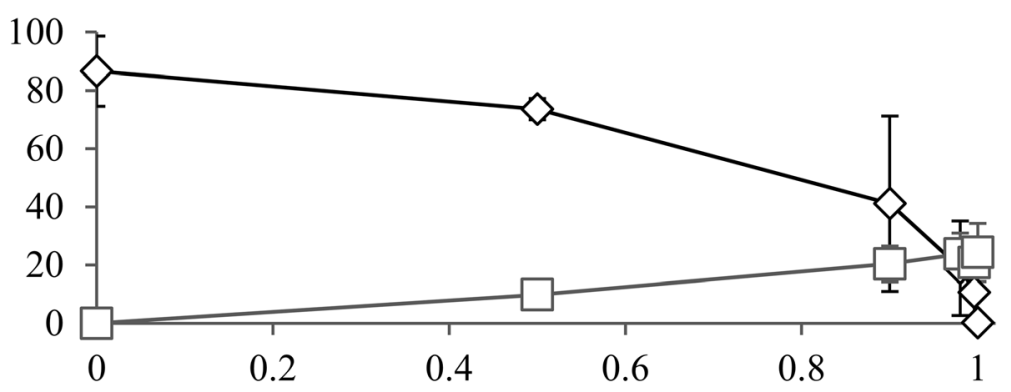

Fig. 2 Proportion (\% of total intermediates and 18:2n-6) of cis-9, trans-11 CLA + trans-11 18:1 (diamond) and trans-10, cis-12 CLA + trans-10 18:1 (square) after a $24 \mathrm{~h}$ incubation period in relation to the relative biomass of Cutibacterium acnes DSM 1897 in the inoculum. The initial concentration of $18: 2 n-6$ was $40 \mu \mathrm{g} / \mathrm{mL}$. a, control medium; $\mathbf{b}$, low pH medium, control medium with pH adjusted to 5.5; c, DHA-enriched (docosahexaenoic acid) medium, control medium containing $40 \mu \mathrm{g} / \mathrm{mL}$ of $22: 6 n-3$

intermediates have not yet been unambiguously identified. Therefore, 28 different rumen species were incubated individually in the presence of $40 \mu \mathrm{g} / \mathrm{mL} 18: 3 n-3$ or 18:2n-6 (Exp. 1). If available, bacterial strains isolated from the rumen were used, if not, strains originated from the gut or feces from other animals or from human tissue were used. Since biohydrogenation of polyunsaturated FA is species and strain specific (e.g. [10, 31]), different strains were included for each genus if available. As Butyrivibrio spp. are well-known trans-11 producers (e.g. [6, 9]), two Butyrivibrio species, i.e. B. fibrisolvens and $B$. proteoclasticus, were included in this study as a negative control. In accordance with previous reports
[12, 32], B. fibrisolvens formed trans-11 intermediates from 18:3n-3 (i.e. cis-9, trans-11, cis-15 CLnA and trans-11, cis15 18:2) and 18:2n-6 (i.e. trans-11 18:1). B. proteoclasticus additionally produced 18:0 from $18: 2 n-6$ as observed by Maia et al. [8].

Other genera included in experiment 1 were selected based on results from the literature. Previous in vivo experiments in our laboratory $[18,21]$ revealed the potential contribution of genera Acidaminococcus, Bifidobacterium and Lactobacillus in the ruminal formation of trans-10 intermediates. Moreover, previous in vitro experiments with Lactobacillus spp., isolated from cheese [12] or from the human intestine [33], showed trans-10, cis-12 CLA 
Table 5 Proportion (\% of total intermediates and 18:2n-6) of 18:2n-6 and its biohydrogenation intermediates after $24 \mathrm{~h}$ of incubation under different growth conditions ${ }^{\text {a }}$ with different biomass ratios of Butyrivibrio fibrisolvens D1 to Propionibacterium acnes DSM 1897 in the inoculum (Exp. 4)

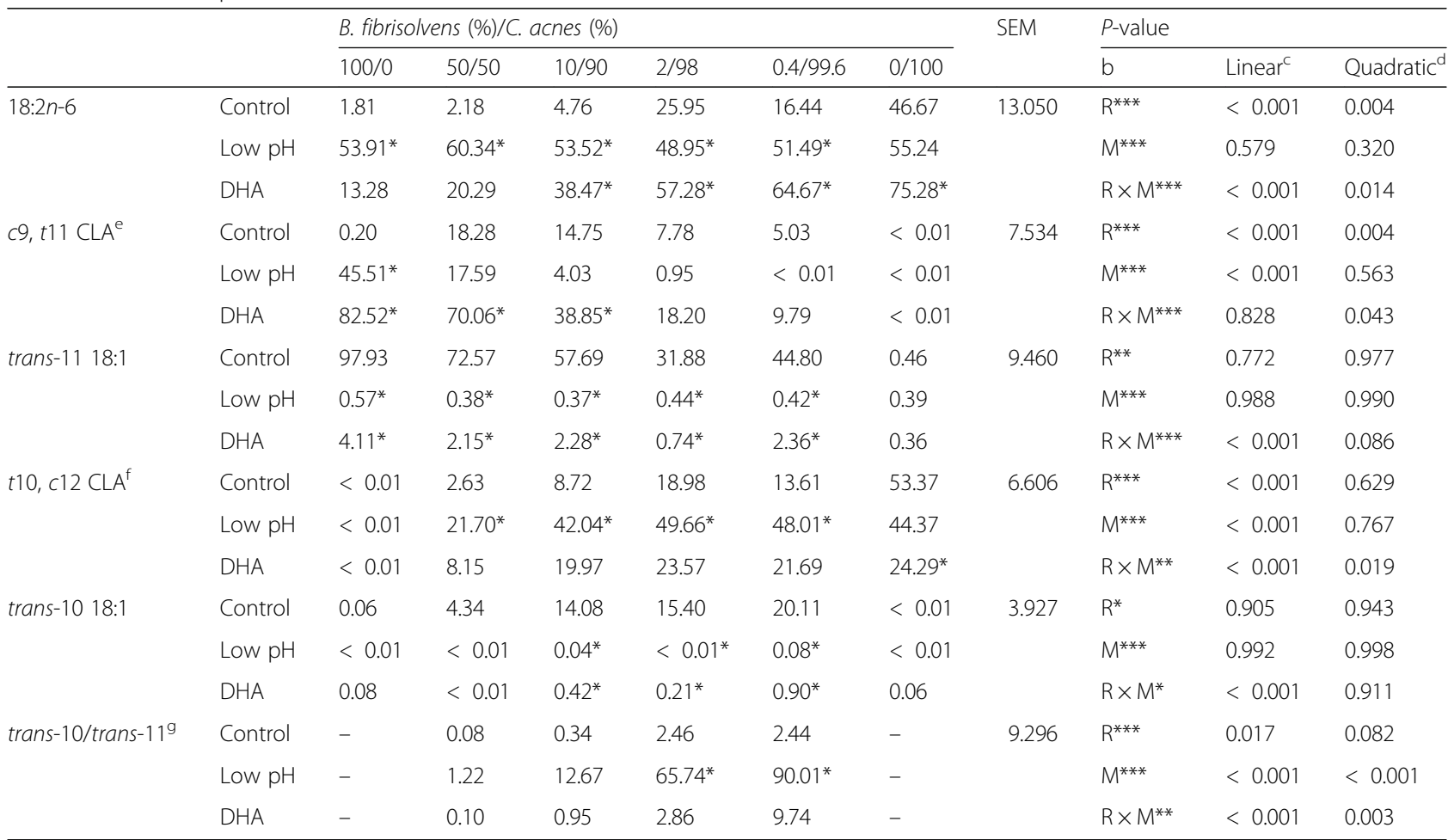

${ }^{a}$ Low $\mathrm{pH}$, control medium with $\mathrm{pH}$ adjusted to 5.5; DHA (docosahexaenoic acid), control medium containing $40 \mu \mathrm{g} / \mathrm{mL}$ of $22: 6 n-3$; all growth media contained $40 \mu \mathrm{g} / \mathrm{mL}$ of $18: 2 n-6$

${ }^{\mathrm{b}} \mathrm{R}$, effect of ratio of $B$. fibrisolvens to $C$. acnes in the inoculum; $\mathrm{M}$, effect of growth medium; ${ }^{*} 0.01 \leq P<0.05$; ${ }^{* *} 0.001 \leq P<0.01$; ${ }^{* * *} P<0.001$

' Linear effect of $R$ within each growth medium

d Quadratic effect of $R$ within each growth medium

e c9, $t 11$ CLA, cis-9, trans-11 conjugated linoleic acid

${ }^{\mathrm{f}} t 10, c 12 \mathrm{CLA}$, trans-10, cis-12 conjugated linoleic acid

${ }^{g}$ Ratio of trans-10 intermediates (trans-10, cis-12 CLA + trans-10 18:1) to trans-11 intermediates (cis-9, trans-11 CLA + trans-11 18:1)

* Means differ $(P<0.05)$ from the control growth medium within the same ratio

-: The ratio is not relevant for mono-cultures as trans-11 and trans-10 are produced exclusively with mono-cultures of $B$. fibrisolvens and $C$. acnes, respectively

formation from $18: 2 n-6$ by various species of this genus. Nevertheless, from the three genera investigated here, only Bifidobacterium metabolized $18: 3 n-3$ and $18: 2 n-6$ in the current study, and no trans-10 intermediates were formed. In contrast to other studies [11,34,35], in which formation of trans-11 intermediates was observed by Bifidobacterium spp., the two Bifidobacterium species used in our study produced hydroxy FA after $24 \mathrm{~h}$ of incubation.

Originally, four other bacteria (i.e. Carnobacterium divergens 66, Carnobacterium maltaromaticum MX 5, Dialister invisus E7.25 and Dialister pneumosintes Cal 4692-1-74) were also included in this study because of positive correlations between their rumen or buccal abundance and trans-10 intermediates in the rumen, milk or blood [18, 21, 25]. However, as those bacteria did not grow in our growth medium, which reflects the rumen conditions, it was not possible to investigate their potential metabolism of $18: 3 n-3$ and $18: 2 n-6$ in the current study. To our knowledge, rumen isolates from the genera Carnobacterium and Dialister are currently not available. Isolation of those genera from the rumen could thus resolve this issue.

Milk fat depression or situations associated with greater trans-10 accumulation are often associated with increased ruminal abundance of $M$. elsdenii [18, 19, 21], Streptococcus spp. and Selenomonas spp. (e.g. [26, 27]), suggesting that those bacteria are involved in the formation of trans-10 intermediates from $18: 2 n-6$ or $18: 3 n-3$. Indeed, Kim et al. [22] found that two strains of ruminal M. elsdenii (i.e. YJ-4 and T81) converted 18:2n-6 to trans-10, cis-12 CLA. In contrast, neither of the two $M$. elsdenii strains analyzed in the study of Maia et al. [8], i.e. LC1 and T81, formed trans-10, cis-12 CLA. In the current study, fourteen different strains of $M$. elsdenii, including LC1 and T81, were incubated with 18:3n-3 and 18:2n-6. Under the experimental conditions used here, only two strains were able to metabolize $18: 3 n-3$ and $18: 2 n-6$, i.e. $2602 \mathrm{~A}$ and 5052B. Nevertheless, the formed intermediates were mainly hydroxy FA rather than the expected trans-10 intermediates. Those results 


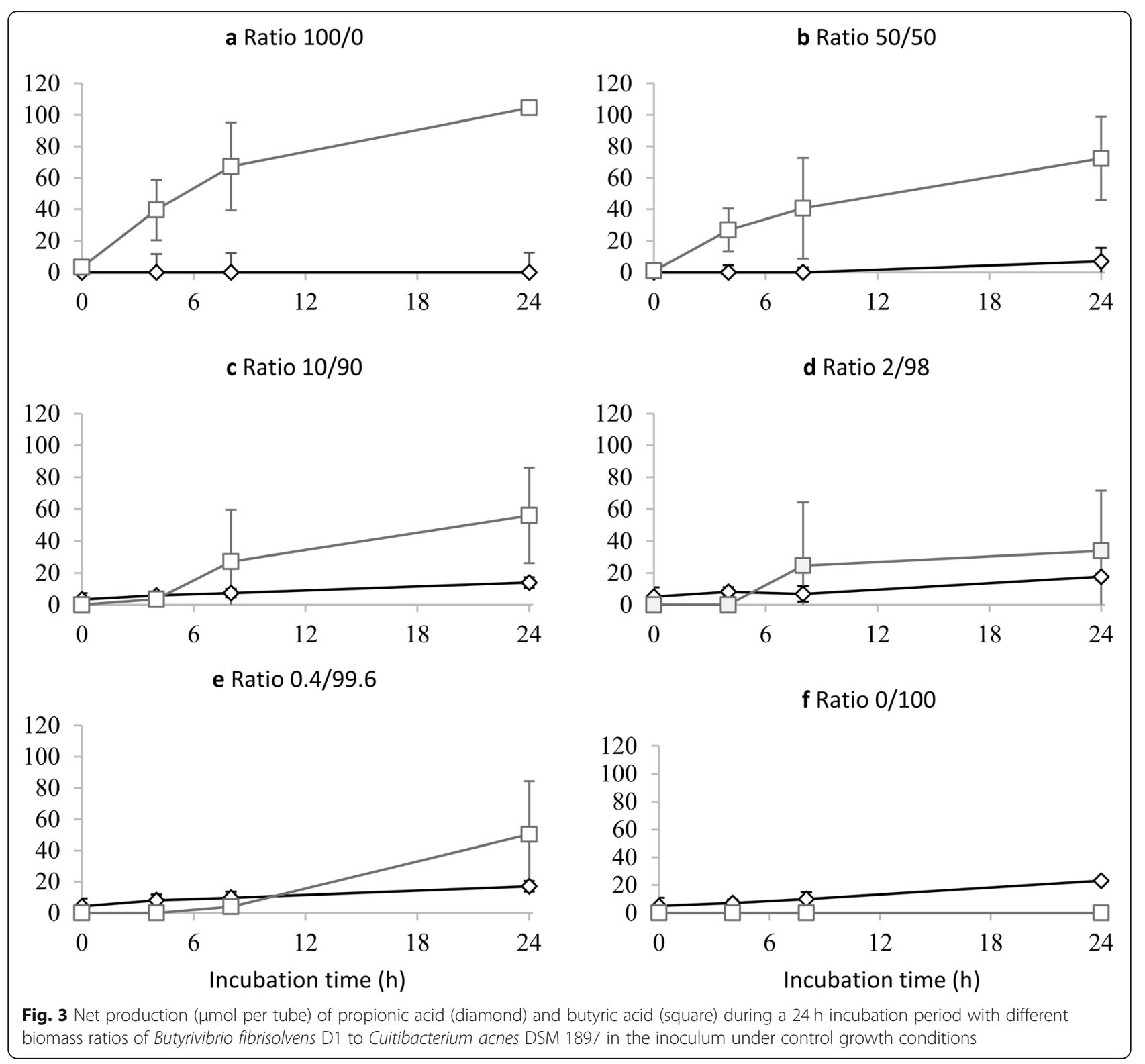

suggest that it is unlikely that $M$. elsdenii directly contributes to the trans-11 to trans-10 shift. The two $S$. ruminantium strains used in this study did not convert 18:3n-3 or $18: 2 n-6$. In contrast, both $S$. equinus and $S$. gallolyticus converted 18:3n-3 and 18:2n-6, but - as for the two Megasphaera strains - the accumulated FA were identified as hydroxy FA rather than trans-10 intermediates, which is in accordance with Maia et al. [8].

$R$. albus and $C$. acnes were selected based on pure culture studies, which suggested trans-10 production by the respective bacteria. Kemp et al. [23] observed formation of trans-10 18:1 from 18:2n-6 and 18:3n-3 by a strictly anaerobic bacterium isolated from sheep rumen, designated as $R$. albus. However, the strain used in the current study did not convert $18: 3 n-3$ or $18: 2 n-6$. Up till now, no other studies investigated $18: 3 n-3$ or $18: 2 n-6$ metabolism by this particular species. In accordance with Wallace et al. [16] and McKain et al. [7], C. acnes produced trans-10, cis-12 CLA from 18:2n-6. When incubated with $18: 3 n-3, C$. acnes also formed a trans-10 intermediate, i.e. trans-10, cis-12, cis-15 CLnA, which was not observed by Maia et al. [36] using the same strain. The isomerase isolated from $C$. acnes showed the capablilty of converting $18: 3 n-3$ to CLnA isomers with trans-11,trans-13,cis-15 CLn as the main product with trace amounts of trans-10,cis-12,cis-15-CLn [37]. In the study of Alves and Bessa [38] trans-10, cis-15 was observed in rumen contents and they suggested that it may have originated from trans-10, cis-12, cis-15 CLnA. However, in their study, the incubation of $18: 3 n-3$ with 
rumen contents resulted in too limited amounts of $\Delta 10$, 12,15-CLnA to conclude on possible pathways. Indeed, Zenad et al. [39] also reported similar observation: the trans-10 shift when incubating 18:2n-6 with ruminal fluids from cows receiving a high-starch plus oil diet or with high starch substrate, while no trans-10 shift was observed when $18: 3 n-3$ was incubated with this rumen fluid. This suggests that 18:3n-3 may be less important precursor of the trans-10 shift.

Besides C. acnes, none of the investigated bacteria converted $18: 2 n-6$ or $18: 3 n-3$ to trans-10 intermediates. However, some bacteria produced hydroxy FA, i.e. $B$. adolescentis, B. pseudolongum, S. equinus, S. gallolyticus, and M. elsdenii 2602A and 5052B. According to Devillard et al. [29], 10-OH cis-12 18:1 might be converted to cis-9, trans-11 CLA by a mixed community of bacteria originating from the hindgut. As such, we further investigated whether the formed hydration products are converted to trans-10 intermediates by mixed rumen inoculum (Exp. 5). Nevertheless, none of the produced hydroxy FA were converted to trans-10 intermediates by mixed rumen inoculum after $24 \mathrm{~h}$ incubation. The conversion of trans- 10 intermediates to 18:0 is slower than the formation of trans-10 intermediates [40]. Therefore, if any trans-10 intermediates were formed, they would have been visible at the end of the incubation period ( $24 \mathrm{~h}$ ) as long as potential precursors of trans-10 intermediates are present in the incubation, which was the case here.

Contrasting results between different studies using the same bacterial species or strains might indicate that not only the species or strain itself is important, but that also specific conditions, which are apparently still unknown, are needed to produce trans-10 intermediates. Since the majority of the used bacteria are related to ruminal lactate metabolism, which is often altered when feeding high-grain diets [28], we investigated the effect of supplementation of lactate to the medium on the metabolism of 18:2n-6 (Exp. 2). It was hypothesized that lactate-utilizing bacteria would grow better under lactate-enriched conditions and would alter their metabolism and convert 18:2n-6 to trans-10 intermediates. As expected, higher densities and increased accumulation of fermentation products were observed after $24 \mathrm{~h}$ of incubation for most strains of the lactate-utilizing bacteria M. elsdenii and S. ruminantium, which might reflect a better growth of these bacteria under these conditions (Tables S1 and S2). Additionally, the volatile fatty acid profile of $M$. elsdenii changed in lactate-enriched media except for M. elsdenii 5045 and M. elsdenii 5052B: under control conditions, the primary VFA was butyrate, while in lactate-enriched media the primary VFA product was propionate. Nevertheless, this higher density and fermentation activity were not associated with an altered metabolism of 18:2n-6. In contrast, the strain M. elsdenii
5052B showed a lower OD value upon incubation with lactate, which was accompanied by a lower proportion of 18:2n-6 disappearance. The lactate concentration used in the current experiment $(200 \mathrm{mM})$ was considerably higher than the normal range reported in the rumen (9$30 \mathrm{mM})$. This potentially impacted the growth of $M$. elsdenii 5052B negatively. Indeed, the rate of lactate utilization and the lactate degradation pathway of M. elsdenii, depends on the lactate concentration in the media [41]. However, originally, a lower lactate concentration (i.e. 9 $\mathrm{mM}$ ) was used (data not shown). As bacterial growth remained unchanged (based on OD600), it was decided to supply lactate according to the growth medium specifications for M. elsdenii in Weimer and Moen [42].

Since trans-11 18:1 was partly isomerized to trans-10 18:1 by mixed cultures in vitro in the study of Laverroux et al. [30], the studied bacteria were additionally incubated in the presence of $40 \mu \mathrm{g} / \mathrm{mL}$ trans-11 18:1 (Exp. 1). Nevertheless, none of the studied bacteria isomerized trans-11 18:1 to trans-10 18:1. Trans-11 18:1 was only converted by $B$. proteoclasticus to yield 18:0, in accordance with McKain et al. [7].

In summary, of the 28 studied rumen bacteria, only $C$. acnes was found to have the metabolic ability to produce trans-10 intermediates from 18:3n-3 or 18:2n-6 under the studied circumstances. Nevertheless, the relevance of this species in ruminal trans-10 formation is questionable given its very low rumen abundance [14, 18], although bacterial activity and kinetics of its $18: 2 n-6$ and 18:3n-3 conversion might play a role besides abundance. Therefore, its biohydrogenation of 18:2n-6 was further investigated and compared with the well-known trans11 producer B. fibrisolvens (Exp. 3 and 4). Furthermore, the effect of different in vitro conditions was investigated (Exp. 3 and 4). Although it could have been of more interest to use a strain originating from the rumen for those experiments, a rumen strain of $C$. acnes is not commercially available and the one isolated by the group of Wallace [16] had been lost (Wallace R. J., personal communication). Nevertheless, in their comparative study [16], similar production rates of trans-10, cis-12 CLA were observed by C. acnes DSM 1897 and C. acnes G449, which was isolated from the rumen.

\section{Assessing the potential importance of $C$. acnes in the trans-11 to trans-10 shift}

B. fibrisolvens in mono-culture (Exp. 3) converted 18:2n6 rapidly (i.e. within the first $8 \mathrm{~h}$ ) to cis-9, trans-11 CLA, which was then further transformed to trans-11 18:1. No trans-10, cis-12 CLA or 18:0 was formed by this bacterium, which is in accordance with previous reports $[7,13]$. The conversion of 18:2n-6 to trans-10, cis- 12 CLA by mono-cultures of $C$. acnes (Exp. 3) was five times slower than the conversion by $B$. fibrisolvens, which might be 
related to the slower growth of this bacterial species under the studied circumstances, as measured by the $\mathrm{OD}_{600}$ (data not shown). The lack of trans-10 18:1 and 18:0 formation by this bacterium indicates that trans-10, cis- 12 CLA is the end product of 18:2n-6 metabolism by $C$. acnes, which is in accordance with a report of McKain et al. [7]. The presence of trans-10 18:1 in co-cultures of these bacterial species (Exp. 4) suggests the possibility of B. fibrisolvens being able to metabolize trans-10, cis-12 CLA. Indeed, Kepler et al. [6] and McKain et al. [7] showed that the reductase of pure cultures of B. fibrisolvens is not highly specific and can convert trans-10, cis-12 CLA to trans-10 18:1. Nevertheless, the formation rate of trans-10 18:1 was lower compared with the formation rate of trans-11 18:1, which is also in agreement with the observations of Kepler et al. [6].

As a consequence of the slower conversion of 18:2n-6 to trans-10, cis-12 CLA compared with the conversion to cis-9, trans-11 CLA, increasing biomass of $C$. acnes in the inoculum in the co-culture experiment (Exp. 4) resulted in an increase in residual 18:2n-6. This confirms the results with mono-cultures of B. fibrisolvens and C. acnes (Exp. 3), which implies that there was no interaction between both bacterial species. The increase in residual 18:2n-6 was, however, more pronounced when $C$. acnes was predominantly present in the initial inoculum. This suggests that $B$. fibrisolvens was responsible for much of the disappearance of 18:2n-6, even when the latter was not the most abundant bacterium initially present. This can be confirmed as cis-9, trans- 11 CLA, trans-11 18:1, and trans-10 18:1 production was observed in those co-cultures where $C$. acnes was predominantly present initially. These FA were not detected in monocultures of C. acnes (Exp. 3), indicating they were produced by $B$. fibrisolvens. Additionally, both propionate and butyrate production was observed in those co-cultures. The production of these metabolites could be used as an indication for the growth or activity of $B$. fibrisolvens and $C$. acnes in the co-cultures of experiment 4, as mono-cultures of these bacterial species (Exp. 3) produced butyric acid and propionic acid, respectively. Even when B. fibrisolvens was hardly present initially, this bacterial species started to grow and was getting important after 4 to $8 \mathrm{~h}$ as suggested by the increase in butyrate concentrations.

The results of this study show that an increasing relative biomass of $C$. acnes results in an increase in the ratio of trans-10 to trans-11 intermediates, and that relatively large biomass proportions of C. acnes (>90\%) in the inoculum are necessary to induce a trans-11 to trans-10 shift under control growth conditions investigated here. This might indicate that the trans-10 shift, as observed in vivo, is not merely a reflection of the relative amounts of cis-9, trans-11 CLA and trans-10, cis-12 CLA producers. Indeed, B. fibrisolvens are by far the most abundant biohydrogenating bacteria in the rumen among the cultured bacteria [43], and the relative amount of $C$. acnes is an order of magnitude lower, even under rumen conditions with a trans-10/trans-11 ratio of $0.82[14,22]$. Perhaps, $C$. acnes behaves in a different way in the rumen as compared to the in vitro observations in the current experiment. As we observed higher relative proportions of $C$. acnes in the oral cavity compared to the rumen in a previous experiment [21], trans-10 formation might perhaps partly take place in the cow's mouth. Nevertheless, further research is required to confirm this hypothesis. Alternatively, the contribution of $C$. acnes to the trans-10 shift observed in the rumen might be limited and other bacterial species could be responsible for the production of trans-10, cis-12 CLA in vivo, which might be more competitive with $B$. fibrisolvens (higher growth rate, higher rate of CLA formation, ...).

Because the trans-11 to trans-10 shift is described as occurring when rumen $\mathrm{pH}$ is low or when marine oils are fed [5], we hypothesized that a low $\mathrm{pH}$ or the addition of DHA to the medium would inhibit the formation of cis-9, trans11 CLA and/or stimulate the formation of trans-10, cis-12 CLA. However, in this study, a low $\mathrm{pH}$ or the addition of DHA reduced the in vitro disappearance of $18: 2 n-6$ by mono-cultures of both B. fibrisolvens and C. acnes (Exp. 3), but the inhibitory effect of low pH or DHA was smaller for C. acnes (14\% reduction) compared to B. fibrisolvens (52\% reduction). Similarly, a low pH or DHA was also more detrimental to butyrate production by $B$. fibrisolvens than to propionate production by $C$. acnes. Indeed, Choi et al. [44] suggested that trans-10, cis-12 CLA-producing rumen bacteria may be more acid-tolerant than cis-9, trans-11 CLA-producing rumen bacteria. Probably, they may also be more tolerant to the presence of polyunsaturated FA, in this case DHA. Supportive of this is the fact that $P$. freudenreichii seems to be more tolerant to higher concentrations of 18:2n-6 [45] compared to B. fibrisolvens [46]. However, this tolerance seems to be strain specific [45]. This higher tolerance of trans-10, cis-12 CLA producers to polyunsaturated FA was also observed in the experiment of Shingfield et al. [14], in which dietary supplementation of fish oil decreased the relative abundance of B. fibrisolvens + Pseudobutyrivibrio spp. and tended to increase the amount of $C$. acnes in omasal content of dairy cows.

Although the inhibitory effect of low $\mathrm{pH}$ or DHA was smaller for trans-10, cis-12 CLA compared to cis-9, trans-11 CLA formation, the absolute amounts of cis-9, trans-11 CLA formed by mono-cultures of $B$. fibrisolvens were still higher than trans-10, cis-12 CLA formed by mono-cultures of $C$. acnes (Exp. 3). Nevertheless, a low $\mathrm{pH}$ or the addition of DHA to the medium reduced the relative biomass of $C$. acnes needed at inoculation to induce a trans-11 to trans-10 shift (Exp. 4), with a low $\mathrm{pH}$ reducing this biomass more (50\%) than DHA addition (90\%).

Under the conditions of this study, the results might indicate that diets inducing a low rumen $\mathrm{pH}$ may be more 
provocative for a trans-11 to trans-10 shift compared to supplementation with marine oils. In agreement with this, Toral et al. [47] observed a trans-11 to trans-10 shift in the rumen after dietary supplementation of starch and sunflower oil, whereas this shift was not observed after dietary supplementation of fish oil. Similarly, dietary supplementation of different levels of fish oil did not result in a trans-11 to trans-10 shift in the experiment of Shingfield et al. [14], which was also the case in the experiment of Zhu et al. [48] after dietary supplementation of DHAenriched microalgae. In contrast to this, dietary supplementation of DHA-enriched microalgae did induce a trans-11 to trans-10 shift in the experiment of Boeckaert et al. [49]. Probably, the effect of DHA supplementation on rumen biohydrogenation is related to its supplementation level [45], and potentially also to the ruminant species to which marine products are supplemented [50].

Supplementation of marine lipids results in the accumulation of $18: 1$ isomers in vivo $[49,51]$, both of trans-11 18 : 1 and trans-10 18:1 (e.g. [14, 48, 51]). This accumulation is mostly due to inhibition of the final step of biohydrogenation to 18:0. Inhibition of the transformation of cis-9, trans-11 CLA to trans-11 18:1 by B. fibrisolvens upon DHA supplementation to the medium is thus surprising, and might indicate that the contribution of this species to the in vivo formation of trans-11 18:1 is less important than originally assumed. Alternatively, B. fibrisolvens behaves in a different way in the rumen as compared with the in vitro observations in the current experiment. The media used for the current experiments contained centrifuged rumen fluid which lacked particles. According to Harfoot et al. [52], rumen fluid particles can reduce the toxicity of PUFA and stimulate the biohydrogenation.

As differences in biohydrogenation kinetics between different in vitro conditions depended on bacterial species, the results of this experiment should be taken with caution. The high required biomass proportion of $C$. acnes at inoculation to induce a trans-11 to trans-10 shift is potentially not only the result of the specific strains which were used, but the specific in vitro conditions could also have strongly influenced this required proportion. Furthermore, the observed strain specificity further complicates extrapolation of our in vitro results to in vivo conditions.

\section{Conclusions}

Among the bacterial species studied, $C$. acnes was the only bacterium having the metabolic ability to produce trans-10 intermediates from $18: 3 n-3$ and 18:2n-6 under the studied circumstances. Nevertheless, it seems unlikely that $C$. acnes is the only or predominant species involved in the trans-11 to trans-10 shift in vivo. Other bacteria were confirmed or found to produce trans-11 intermediates (i.e. B. fibrisolvens and B. proteoclasticus) or hydroxy FA (i.e. B. adolescentis, B. pseudolongum, S. equinus, $S$. gallolyticus, M. elsdenii 2602A and 5052B), which were not further converted to trans-10 intermediates by mixed rumen inoculum. None of the studied bacteria isomerized trans-11 18:1 to trans-10 18:1, and addition of lactate to the medium did not alter the metabolism of the bacteria to produce trans-10 intermediates. Nevertheless, the results of this study should be taken with caution as not only bacterial but also environmental features influence competition between bacteria. As such, the in vitro conditions might have strongly affected the obtained results. Furthermore, biohydrogenation seems to be very strain specific, which also complicates extrapolation to in vivo conditions.

\section{Methods}

\section{Micro-organisms and growth conditions}

Batch in vitro incubations were established using pure cultures of 28 rumen bacterial species. The provenance of those species is shown in Table 6. If available, bacterial strains isolated from the rumen were used, if not, strains originated from the gut or feces from other animals or from human were used.

Four different growth media were used: i/ control medium ( $\mathrm{pH}=6.70 \pm 0.203$; mean $\pm \mathrm{SD}$ ), ii/ lactate-enriched medium $(\mathrm{pH}=6.82 \pm 0.457$; mean $\pm \mathrm{SD})$, iii/ low $\mathrm{pH}$ medium $(\mathrm{pH}=$ $5.50 \pm 0.005$; mean $\pm \mathrm{SD}$ ), and iv/ docosahexaenoic acid (DHA)-enriched medium ( $\mathrm{pH}=6.62 \pm 0.150$; mean $\pm \mathrm{SD})$. A slightly modified Butyrivibrio medium (medium 704; Deutsche Sammlung von Mikroorganismen and Zellkulturen $\mathrm{GmbH}$, Braunschweig, Germany) was used as a control medium. The modification was based on Jeyanathan et al. [53]). The control medium used in the current set-up contained (per $\mathrm{L}$ ): $90 \mathrm{~mL}$ mineral solution (per $\mathrm{L}$ distilled water, $6 \mathrm{~g} \mathrm{KH}_{2} \mathrm{PO}_{4}, 12 \mathrm{~g} \mathrm{NaCl}, 6 \mathrm{~g}\left(\mathrm{NH}_{4}\right)_{2} \mathrm{SO}_{4}, 1.6 \mathrm{~g} \mathrm{CaCl}_{2} \cdot 2 \mathrm{H}_{2} \mathrm{O}$, $2.5 \mathrm{~g} \mathrm{MgSO}_{4} .7 \mathrm{H}_{2} \mathrm{O}$ ), $150 \mathrm{~mL}$ rumen fluid, $0.3 \mathrm{~g} \mathrm{~K}_{2} \mathrm{HPO}_{4}, 2 \mathrm{~g}$ trypticase peptone, $2 \mathrm{~g}$ yeast extract, $0.5 \mathrm{~mL} \mathrm{Na}$-resazurin solution, $4 \mathrm{~g} \mathrm{Na}_{2} \mathrm{CO}_{3}, 1 \mathrm{~g}$ each of glucose, maltose, cellobiose and starch, and $0.5 \mathrm{~g}$ of L-Cysteine- $\mathrm{HCl}$., Compared with the original basic medium, a volatile FA (VFA) mixture (3.1 $\mathrm{mL} / \mathrm{L})$, hemin $(2 \mathrm{~mL} / \mathrm{L})$ and glycerol $(0.5 \mathrm{~g} / \mathrm{L})$ were omitted from the basic medium (as these substrates are supposed to be present in the rumen fluid), and L-cysteine- $\mathrm{HCl}(0.5 \mathrm{~g} / \mathrm{L})$ was used as the only reducing agent whereas in the original basic medium a mixture (1:1) of L-Cysteine- $\mathrm{HCl}$ and $\mathrm{Na}_{2} \mathrm{~S} .9 \mathrm{H}_{2} \mathrm{O}$ was used. Some further modifications were applied to the preparation of rumen fluid (see further) and the rumen fluid/buffer ratio (20\% rumen fluid instead of $15 \%, v / v)$. The rumen fluid was collected from three adult sheep. These were fitted with a ruminal cannula and were fed grass hay ad libitum and a commercial, pelleted grainbased concentrate $(200 \mathrm{~g} / \mathrm{d})$ twice a day, at $09 \mathrm{~h} 00$ and 17 h00, according to their maintenance requirements. Approximately $0.5 \mathrm{~L}$ of ruminal digesta was collected from each animal just before the morning feeding. The collected rumen fluid was filtered through a sieve with a pore size of 
Table 6 Provenance of the different bacterial strains used in the experiment

\begin{tabular}{|c|c|c|c|c|c|c|c|}
\hline No. & Family & Genus & Species & Strain & Origin & Comments & Source \\
\hline 1 & Acidaminococcaceae & Acidaminococcus & fermentans & VR4 & Pig gut, unknown ${ }^{a}$ & Type strain & DSMZ \\
\hline 2 & Acidaminococcaceae & Acidaminococcus & intestini & ADV 255.99 & $\begin{array}{l}\text { Human peritoneal fluid, } \\
\text { France }\end{array}$ & Type strain & DSMZ \\
\hline 3 & Bifidobacteriaceae & Bifidobacterium & adolescentis & RU 424 & Bovine rumen, unknown ${ }^{a}$ & & DSMZ \\
\hline 4 & Bifidobacteriaceae & Bifidobacterium & pseudolongum & RU224 & Rumen, unknown ${ }^{\mathrm{a}}$ & $\begin{array}{l}\text { Subsp. globosum, } \\
\text { type strain }\end{array}$ & DSMZ \\
\hline 5 & Lachnospiraceae & Butyrivibrio & fibrisolvens & D1 & Bovine rumen, unknown ${ }^{a}$ & Type strain & DSMZ \\
\hline 6 & Lachnospiraceae & Butyrivibrio & proteoclasticus & P18 & Sheep rumen, UK & & Dr. J. Wallace \\
\hline 7 & Lactobacillaceae & Lactobacillus & ruminis & RF1 & Bovine rumen, unknown ${ }^{a}$ & Type strain & DSMZ \\
\hline 8 & Lactobacillaceae & Lactobacillus & ruminis & RF2 & Bovine rumen, unknown ${ }^{a}$ & & DSMZ \\
\hline 9 & Propionibacteriaceae & Propionibacterium & acnes & DSM 1897 & $\begin{array}{l}\text { Acne lesion in human } \\
\text { facial skin, unknown }\end{array}$ & Type strain & DSMZ \\
\hline 10 & Ruminococcaceae & Ruminococcus & albus & 7 & Bovine rumen, unknown ${ }^{a}$ & Type strain & DSMZ \\
\hline 11 & Streptococcaceae & Streptococcus & equinus & Pearl 11 & Cow dung, unknown ${ }^{a}$ & & DSMZ \\
\hline 12 & Streptococcaceae & Streptococcus & gallolyticus & DSM 16831 & Koala feces, Australia & Type strain & DSMZ \\
\hline 13 & Veillonellaceae & Megasphaera & elsdenii & B159 & Cow rumen, USA & & Dr. P. Weimer \\
\hline 14 & Veillonellaceae & Megasphaera & elsdenii & T81 & Cow rumen, USA & & Dr. P. Weimer \\
\hline 15 & Veillonellaceae & Megasphaera & elsdenii & LC1 & Sheep rumen, unknown ${ }^{a}$ & Type strain & DSMZ \\
\hline 16 & Veillonellaceae & Megasphaera & elsdenii & $2602 A$ & Cow rumen, USA & & Dr. P. Weimer \\
\hline 17 & Veillonellaceae & Megasphaera & elsdenii & $3016 B$ & Cow rumen, USA & & Dr. P. Weimer \\
\hline 18 & Veillonellaceae & Megasphaera & elsdenii & $3218 \mathrm{~A}$ & Cow rumen, USA & & Dr. P. Weimer \\
\hline 19 & Veillonellaceae & Megasphaera & elsdenii & $3436 \mathrm{~A}$ & Cow rumen, USA & & Dr. P. Weimer \\
\hline 20 & Veillonellaceae & Megasphaera & elsdenii & 4251 & Cow rumen, USA & & Dr. P. Weimer \\
\hline 21 & Veillonellaceae & Megasphaera & elsdenii & 4257 & Cow rumen, USA & & Dr. P. Weimer \\
\hline 22 & Veillonellaceae & Megasphaera & elsdenii & 4296 & Cow rumen, USA & & Dr. P. Weimer \\
\hline 23 & Veillonellaceae & Megasphaera & elsdenii & 4400 & Cow rumen, USA & & Dr. P. Weimer \\
\hline 24 & Veillonellaceae & Megasphaera & elsdenii & 5045 & Cow rumen, USA & & Dr. P. Weimer \\
\hline 25 & Veillonellaceae & Megasphaera & elsdenii & $5052 B$ & Cow rumen, USA & & Dr. P. Weimer \\
\hline 26 & Veillonellaceae & Megasphaera & elsdenii & 5596 & Cow rumen, USA & & Dr. P. Weimer \\
\hline 27 & Veillonellaceae & Selenomonas & ruminantium & GA-192 & Bovine rumen, USA & $\begin{array}{l}\text { Subsp. ruminantium, } \\
\text { type strain }\end{array}$ & DSMZ \\
\hline 28 & Veillonellaceae & Selenomonas & ruminantium & PC 18 & Bovine rumen, USA & $\begin{array}{l}\text { Subsp. lactilytica, } \\
\text { type strain }\end{array}$ & DSMZ \\
\hline
\end{tabular}

${ }^{\mathrm{a}}$ Unknown, country of origin unknown

$1 \mathrm{~mm}$ and combined. Then, the combined rumen fluid was sterilized by autoclaving for $20 \mathrm{~min}$ at $121^{\circ} \mathrm{C}$. To remove fine particles, the rumen fluid was centrifuged twice, the first time at $14,000 \times g$ and the second time at $20,000 \times g$, each time for $15 \mathrm{~min}$ at $4{ }^{\circ} \mathrm{C}$. The supernatant was stored at $-20^{\circ} \mathrm{C}$ and thawed before use. The lactate-enriched medium was the control medium supplemented with Na-lactate (SigmaAldrich, Diegem, Belgium) to a final concentration of 200 $\mathrm{mM}$ (based on a growth medium specifically used for $M$. elsdenii in Weimer and Moen [42]). Initially, a lower concentration more closely related to potential rumen lactate concentrations (i.e. $9 \mathrm{mM}$ ) was used (data not shown), however, as no differences were observed in $\mathrm{OD}_{600}$, it was decided to use a higher concentration. The low $\mathrm{pH}$ medium was prepared by adding $2 \mathrm{M} \mathrm{HCl}$ solution to the control medium to reduce the $\mathrm{pH}$ from 6.5 to 5.5. The DHAenriched medium was the control medium supplemented with DHA (Nu-Chek Prep, Elysian, MN, USA) to a final concentration of $40 \mu \mathrm{g} / \mathrm{mL}$.

These media were transferred to Hungate-type tubes (16 mm i.d., $125 \mathrm{~mm}$ long; Chemglass Life Sciences, Vineland, NJ, USA). Then, 18:3n-3, 18:2n-6 or trans-11 18:1 (NuChek Prep, Elysian, MN, USA) was added into each tube to a final concentration of $40 \mu \mathrm{g} / \mathrm{mL}$, after which the tubes were closed with screw caps fitted with butyl rubber septa (Chemglass Life Sciences, Vineland, NJ, USA). The tubes 
were then autoclaved $\left(121^{\circ} \mathrm{C}, 20 \mathrm{~min}\right)$ prior to inoculum addition. All preparations and transfers were carried out under continuous flushing of $\mathrm{CO}_{2}$.

The inoculum was harvested from fresh cultures of each strain, grown in modified control medium $(30 \%$ rumen fluid instead of $20 \%$, v/v) for 12 to $36 \mathrm{~h}$, depending on the growth rate of the strain $\left(\mathrm{OD}_{600}=1.26 \pm 0.41\right.$; mean $\pm \mathrm{SD}$ ). After inoculum addition, the tubes were maintained under anaerobic conditions at $39^{\circ} \mathrm{C}$, with intermittent shaking in a batch culture incubator (Edmund Bühler GmbH, Hechingen, Germany). The reactions were stopped at different time points (according to the experimental design) by removing the tubes from the incubator and cooling the tubes in an ice bath. Optical density at $600 \mathrm{~nm}\left(\mathrm{OD}_{600}\right.$; Ultraspec10, Amersham Biosciences Corp., Piscataway, NJ, USA) and pH (Hanna Instruments, Temse, Belgium) were measured, and subsamples were collected for analysis of VFA $(2 \mathrm{~mL})$ and long-chain FA (LCFA; remainder of the tube, i.e. $8 \mathrm{~mL}$ ).

\section{Fatty acid solution}

$22: 6 n-3,18: 3 n-3$ and $18: 2 n-6$ solutions were prepared as a watery FA solution containing either $10 \mathrm{~g} / \mathrm{L} 18: 3 n-3$ or 18 : 2n-6 (control medium, lactate-enriched medium and low

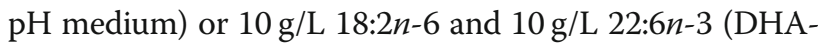
enriched medium), together with $166.7 \mathrm{~mL} / \mathrm{L}$ Tween 20 solution (75 g/L; Sigma-Aldrich, Diegem, Belgium) and $12.5 \mathrm{~mL} / \mathrm{L} 3 \mathrm{M} \mathrm{NaOH}$ (based on Jeyanathan et al. [53]). Tween 20 was added as an emulsifier, whilst $\mathrm{NaOH}$ was added in order to obtain a clear solution. Trans-11 18:1 was dissolved in dimethyl sulfoxide to a final concentration of $10 \mathrm{~g} / \mathrm{L}$. The required amount of each FA solution was added individually to each Hungate tube before autoclaving. The amounts of Tween-80, $\mathrm{NaOH}$ and dimethyl sulfoxide were kept constant in all tubes .

\section{Experimental design}

Table 7 summarizes the experimental setup of the current study. In experiment 1 (Exp. 1), pure cultures of the 28 strains were incubated individually under control growth conditions in the presence of $40 \mu \mathrm{g} / \mathrm{mL} 18: 3 n-3$ or $18: 2 n-6$ or trans-11 $18: 1$ in order to determine their ability to metabolize 18:3n-3, 18:2n-6 and trans-11 18:1. The dose of the FA was decided based on the preliminary in house experiments performed with B. fibrisolvens and C. acnes. To obtain an inoculum size of $5 \%(\mathrm{v} / \mathrm{v}), 0.5 \mathrm{~mL}$ inoculum was added to $9.5 \mathrm{~mL}$ of growth medium. The incubations were stopped after $24 \mathrm{~h}$ to evaluate the disappearance of $18: 3 n-3$, 18:2n-6 or trans-11 18:1 and the intermediates formed.

In a second experiment (Exp. 2), the same 28 strains were incubated individually under lactate-enriched conditions in the presence of $40 \mu \mathrm{g} / \mathrm{mL} 18: 2 n-6$ to assess the influence of lactate on 18:2n-6 metabolism. The inoculum size was $5 \%(\mathrm{v} / \mathrm{v})$ and incubations were stopped after $24 \mathrm{~h}$.
In experiment 3 (Exp. 3), pure cultures of B. fibrisolvens, as a cis-9, trans-11 CLA producer, and C. acnes, as a trans-10, cis-12 CLA producer, were grown separately in three different growth media (control, low $\mathrm{pH}$, and DHAenriched media) in the presence of $40 \mu \mathrm{g} / \mathrm{mL} 18: 2 n-6$ to examine the effect of growth medium on the rate of CLA formation by these bacterial species. In order to obtain an inoculum size of $5 \%(\mathrm{v} / \mathrm{v}), 0.5 \mathrm{~mL}$ inoculum was added to $9.5 \mathrm{~mL}$ growth medium. The incubations were stopped at different time points $(0,2,4,8$ and $24 \mathrm{~h})$ to evaluate the effect of incubation time on CLA formation.

In experiment 4 (Exp. 4), pure cultures of B. fibrisolvens and $C$. acnes were combined to establish different biomass ratios of those species in order to elucidate their effect on the trans-11 to trans-10 shift, in the presence of $40 \mu \mathrm{g} / \mathrm{mL}$ 18:2n-6. Different volumes of the two inoculum sources were mixed together in order to obtain the different ratios. The total inoculum size was $10 \%(\mathrm{v} / \mathrm{v})$. The incubations were stopped at different time points $(0,4,8$ and $24 \mathrm{~h}$ or $24 \mathrm{~h}$ only) depending on the growth medium.

For all experiments mentioned above, each treatment was performed in quadruplicate (analytical replicates). These quadruplicates were spread into duplicates, which were performed on different days. For each quadruplicate, the inoculum was grown separately. On each day, blank samples, containing distilled water as inoculum, were added in duplicate to measure the initial amount of VFA and LCFA before the incubation.

\section{In vitro incubation with mixed rumen inoculum (Exp. 5)}

When $18: 3 n-3$ or $18: 2 n-6$ was converted to a hydroxy FA after $24 \mathrm{~h}$ of incubation by the pure culture in experiment 1, a follow-up incubation was performed to elucidate whether the formed intermediate is further converted to trans-10 intermediates by mixed rumen inoculum (Exp. 5). As sheep seem to be less vulnerable to situations associated with a trans-10 shift compared with cows (e.g. [54]), cow rumen inoculum was used as donor of rumen microbes for this in vitro incubation. The rumen fluid was collected from three lactating HolsteinFriesian dairy cows (analytical replicates), each fitted with a ruminal cannula. The cows were fed a basal diet consisting of maize silage and grass silage (50/50, w/w DM basis) ad libitum, supplemented with a standard concentrate according to their milk production. Samples of ruminal digesta were collected from each animal just before the morning feeding, after which it was filtered through a sieve with a pore size of $1 \mathrm{~mm}$ under continuous flushing with $\mathrm{CO}_{2}$. The filtered rumen fluid was mixed with a bicarbonate/phosphate buffer (rumen fluid/buffer, $1 / 4 \mathrm{v} / \mathrm{v}$ ) containing $0.2125 \mathrm{~g}$ glucose, 0.2125 g cellobiose, $0.1875 \mathrm{~g}$ xylose, $0.1875 \mathrm{~g}$ arabinose, $1.25 \mathrm{~g}$ acid casein hydrolysate, $1.25 \mathrm{~g}$ peptone, $1.25 \mathrm{~g}$ yeast extract, $2.685 \mathrm{~g} \mathrm{Na}_{2} \mathrm{HPO}_{4} \cdot 12 \mathrm{H}_{2} \mathrm{O}, 1.1625 \mathrm{~g} \mathrm{KH}_{2} \mathrm{PO}_{4}, 0.093$ 
Table 7 Overview of the different pure culture experiments conducted

\begin{tabular}{|c|c|c|c|c|c|}
\hline & Bacterium $^{a}$ (inoculum size, v/v) & $\begin{array}{l}\text { Ratio of } B \text {. fibrisolvens } \\
(\%) / C \text {. acnes }(\%) \text { in } \\
\text { the inoculum }\end{array}$ & Growth medium ${ }^{c}$ & $\begin{array}{l}\text { Fatty acid } \\
\text { substrate }^{d}\end{array}$ & Incubation period (h) \\
\hline \multirow[t]{3}{*}{ Exp. 1} & \multirow{3}{*}{$\begin{array}{l}28 \text { strains individually } \\
\text { (Table 1) (5\%) }\end{array}$} & \multirow[t]{3}{*}{ NA } & \multirow[t]{3}{*}{ Control } & $18: 3 n-3$ & 24 \\
\hline & & & & $18: 2 n-6$ & 24 \\
\hline & & & & trans-11 18:1 & 24 \\
\hline Exp. 2 & $\begin{array}{l}28 \text { strains individually } \\
\text { (Table 1) (5\%) }\end{array}$ & NA & Lactate & $18: 2 n-6$ & 24 \\
\hline \multirow[t]{6}{*}{ Exp. 3} & \multirow[t]{3}{*}{ B. fibrisolvens (5\%) } & \multirow[t]{3}{*}{$100 / 0$} & Control & $18: 2 n-6$ & $0,2,4,8$, and 24 \\
\hline & & & Low pH & $18: 2 n-6$ & $0,2,4,8$, and 24 \\
\hline & & & DHA & $18: 2 n-6$ & $0,2,4,8$, and 24 \\
\hline & \multirow[t]{3}{*}{ C. acnes (5\%) } & \multirow[t]{3}{*}{$0 / 100$} & Control & $18: 2 n-6$ & $0,2,4,8$, and 24 \\
\hline & & & Low pH & $18: 2 n-6$ & $0,2,4,8$, and 24 \\
\hline & & & DHA & $18: 2 n-6$ & $0,2,4,8$, and 24 \\
\hline \multirow[t]{18}{*}{ Exp. 4} & \multirow[t]{3}{*}{ B. fibrisolvens (10\%) } & \multirow[t]{3}{*}{$100 / 0$} & Control & $18: 2 n-6$ & $0,4,8$, and 24 \\
\hline & & & Low pH & $18: 2 n-6$ & 24 \\
\hline & & & DHA & $18: 2 n-6$ & 24 \\
\hline & \multirow{3}{*}{$\begin{array}{l}\text { B. fibrisolvens (5\%) } \\
\text { and C. acnes (5\%) }\end{array}$} & \multirow[t]{3}{*}{$50 / 50$} & Control & $18: 2 n-6$ & $0,4,8$, and 24 \\
\hline & & & Low pH & $18: 2 n-6$ & 24 \\
\hline & & & DHA & $18: 2 n-6$ & 24 \\
\hline & \multirow{3}{*}{$\begin{array}{l}\text { B. fibrisolvens (1\%) } \\
\text { and C. acnes (9\%) }\end{array}$} & \multirow[t]{3}{*}{$10 / 90$} & Control & $18: 2 n-6$ & $0,4,8$, and 24 \\
\hline & & & Low pH & $18: 2 n-6$ & 24 \\
\hline & & & DHA & $18: 2 n-6$ & 24 \\
\hline & \multirow{3}{*}{$\begin{array}{l}\text { B. fibrisolvens (0.2\%) } \\
\text { and C. acnes }(9.8 \%)\end{array}$} & \multirow[t]{3}{*}{ 2/98 } & Control & $18: 2 n-6$ & $0,4,8$, and 24 \\
\hline & & & Low pH & $18: 2 n-6$ & 24 \\
\hline & & & DHA & $18: 2 n-6$ & 24 \\
\hline & \multirow{3}{*}{$\begin{array}{l}\text { B. fibrisolvens }(0.04 \%) \\
\text { and C. acnes }(9.96 \%)\end{array}$} & \multirow[t]{3}{*}{$0.4 / 99.6$} & Control & $18: 2 n-6$ & $0,4,8$, and 24 \\
\hline & & & Low pH & $18: 2 n-6$ & 24 \\
\hline & & & DHA & $18: 2 n-6$ & 24 \\
\hline & \multirow[t]{3}{*}{ C. acnes (10\%) } & \multirow[t]{3}{*}{$0 / 100$} & Control & $18: 2 n-6$ & $0,4,8$, and 24 \\
\hline & & & Low pH & $18: 2 n-6$ & 24 \\
\hline & & & DHA & $18: 2 n-6$ & 24 \\
\hline
\end{tabular}

\footnotetext{
${ }^{a}$ Fresh cultures, grown in modified control medium ( $30 \%$ rumen fluid instead of $\left.20 \%, v / v\right)$ for 12 to $36 \mathrm{~h}$, depending on the growth rate of the strain $\left(\mathrm{OD}_{600}=\right.$ $1.26 \pm 0.41$; mean $\pm S D$ ), were used as inoculum

${ }^{b} \mathrm{NA}$, not applicable

' Lactate, control medium supplemented with $200 \mathrm{mM}$ Na-lactate; low pH, control medium with pH adjusted to 5.5; DHA (docosahexaenoic acid), control medium containing $40 \mu \mathrm{g} / \mathrm{mL}$ of $22: 6 n-3$

d The initial amount of fatty acid was $40 \mu \mathrm{g} / \mathrm{mL}$
}

g $\mathrm{MgCl}_{2} \cdot 6 \mathrm{H}_{2} \mathrm{O}$, $6.555 \mathrm{~g} \mathrm{NaHCO}_{3}$ and $0.75 \mathrm{~g} \mathrm{NH}_{4} \mathrm{HCO}_{3}$ per liter of distilled water $(\mathrm{pH} \approx 6.8)$.

To assess further metabolism of hydroxy FA, these FA were produced in incubations with the bacterial strains identified during the first part of the study to biohydrate either $18: 3 n-3$ or $18: 2 n-6$ using the same methodology as described above (Exp. 1). Of each treatment (strain $x$ $18: 3 n-3 / 18: 2 n-6), 10$ Hungate tubes were incubated for $24 \mathrm{~h}$ starting from the same inoculum. Then, those tubes were pooled, $25 \mathrm{~mL}$ was transferred into $120-\mathrm{mL}$ incubation flasks, after which it was freeze-dried. The amounts of hydroxy FA, accumulating at the end of the initial incubation were quantified for each bacterial strain. Into the flasks containing $25 \mathrm{~mL}$ of the freezedried pure culture medium with the hydroxy FA, a 25 $\mathrm{mL}$ rumen fluid/buffer mixture was added. The cultures were maintained under anaerobic conditions at $39^{\circ} \mathrm{C}$, with intermittent shaking in a batch culture incubator (Edmund Bühler GmbH, Hechingen, Germany). Reactions were stopped after $24 \mathrm{~h}$ of incubation by cooling in 
an ice bath, after which pH (Hanna Instruments, Temse, Belgium) was measured and subsamples were collected for analysis of VFA $(2 \mathrm{~mL})$ and LCFA $(10 \mathrm{~mL})$.

\section{Chemical analysis and calculations}

For VFA analysis, $2 \mathrm{~mL}$ of incubation medium was collected and acidified immediately with $200 \mu \mathrm{L}$ of formic acid, which contained an internal standard (10 mg of 2ethylbutyric acid (Sigma-Aldrich, Diegem, Belgium) per $\mathrm{mL}$ of formic acid). After centrifugation $\left(15 \mathrm{~min}\right.$ at $4{ }^{\circ} \mathrm{C}$ and $31,000 \times g$ ), the supernatant was filtered and transferred into a $1.5 \mathrm{~mL}$ glass vial. Samples were stored at $4{ }^{\circ} \mathrm{C}$ until analysis using a gas chromatograph (HP7890A; Agilent Technologies, Diegem, Belgium) equipped with a Supelco Nukol capillary column $(30 \mathrm{~m} \times 0.25 \mathrm{~mm}$ i.d. $\times 0.25 \mu \mathrm{m}$ thickness; Sigma-Aldrich, Diegem, Belgium) and a flame ionization detector. The temperature program was as follows: $120^{\circ} \mathrm{C}$ at injection for $0.2 \mathrm{~min}$, increased at $10^{\circ} \mathrm{C} /$ min to $180^{\circ} \mathrm{C}$, and kept at this temperature for $3 \mathrm{~min}$; injector temperature: $250^{\circ} \mathrm{C}$; detector temperature: $255^{\circ} \mathrm{C}$. For this temperature program, $0.3 \mu \mathrm{L}$ was injected with a split/split less ratio of 25:1 using $\mathrm{H}_{2}$ as carrier gas at 0.8 $\mathrm{mL} / \mathrm{min}$. Volatile FA peaks were identified based on their retention times, compared to external standards (Sigma-Aldrich, Diegem, Belgium). Net production of VFA was calculated by subtracting the amount in the incubation medium before incubation from the amount after incubation.

For analysis of LCFA, incubation medium $(8 \mathrm{~mL}$ for pure culture incubations, Exp. 1-4; and $10 \mathrm{~mL}$ for the mixed community incubations, Exp. 5) was collected in glass tubes at $0 \mathrm{~h}$ and after the incubation, and immediately frozen at $-20{ }^{\circ} \mathrm{C}$ and freeze-dried prior to further analysis. Fatty acids were methylated as described by Dewanckele et al. [18]. Briefly, toluene (2 mL) containing the internal standard (21:0; Sigma-Aldrich, Diegem, Belgium) and methanolic $\mathrm{NaOH}(2 \mathrm{~mL} ; 0.5 \mathrm{M})$ were added and the mixture was incubated at $70^{\circ} \mathrm{C}$ for 60 min. This was followed by $30 \mathrm{~min}$ at $50^{\circ} \mathrm{C}$ after addition of methanolic $\mathrm{HCl}(3 \mathrm{~mL})$, prepared by dissolving acetyl chloride in methanol $(5 / 1, v / v)$. Fatty acid methyl esters (FAME) were extracted with hexane. Analysis of FAME was carried out using a gas chromatograph (HP7890A; Agilent Technologies, Diegem, Belgium) equipped with an SP-2560 capillary column $(75 \mathrm{~m} \times 0.18 \mathrm{~mm}$ i.d. $\times 0.14 \mu \mathrm{m}$ thickness; Supelco Analytical, Bellefonte, PA, USA) and a flame ionization detector. The temperature program was as follows: initially $70^{\circ} \mathrm{C}$ for $2 \mathrm{~min}$, increasing by $15^{\circ} \mathrm{C} /$ min to $150^{\circ} \mathrm{C}$, followed by a second increase at $1^{\circ} \mathrm{C} / \mathrm{min}$ up to $165^{\circ} \mathrm{C}$ and holding for $12 \mathrm{~min}$, followed by a third increase at $2{ }^{\circ} \mathrm{C} / \mathrm{min}$ to $170{ }^{\circ} \mathrm{C}$, held at $170{ }^{\circ} \mathrm{C}$ for $5 \mathrm{~min}$, increased at $5{ }^{\circ} \mathrm{C} / \mathrm{min}$ to $215^{\circ} \mathrm{C}$, and held at $215^{\circ} \mathrm{C}$ for 20 min. Inlet and detector temperatures were $250^{\circ} \mathrm{C}$ and $255^{\circ} \mathrm{C}$, respectively. Injection volume was $1 \mu \mathrm{L}$ with a split ratio of 25:1. Hydrogen was used as the carrier gas at a flow rate of $1 \mathrm{~mL} / \mathrm{min}$.

Peaks were routinely identified based on retention time comparisons with commercial mixtures of methyl ester standards (GLC463, cis-9, trans-11 CLA and trans-10, cis12 CLA; Nu-Chek Prep, Elysian, MN, USA). Methyl esters of formed intermediates not contained in commercially available standards were identified by GC-MS analysis of 4, 4-dimethyloxazoline (DMOX) derivatives, prepared from FAME by using a modified procedure [55]. Briefly, FAME were converted into DMOX derivatives with $250 \mathrm{mg} 2$ amino-2-methyl-1-propanol under a nitrogen atmosphere at $175^{\circ} \mathrm{C}$ overnight. DMOX derivatives were extracted twice with diethyl ether/hexane $(1 / 1, \mathrm{v} / \mathrm{v})$ and sodium chloride saturated water. The organic layer was dried with anhydrous sodium sulphate for $1 \mathrm{~h}$, followed by evaporation until dry under nitrogen. The DMOX derivatives were dissolved in hexane. Impact ionization spectra of DMOX derivatives were obtained using a gas chromatograph equipped with a quadrupole mass detector (Trace DSQ II, Thermo Scientific, Waltham, MA, USA). Mass spectra were recorded under an ionization voltage of $70 \mathrm{eV}$ (EI+ mode), using Xcalibur software (version 1.4 SR1) for data acquisition and processing. The column was a SLB-IL 60 capillary column $(30 \mathrm{~m} \times 0.25 \mathrm{~mm}$ i.d. $\times 0.2 \mu \mathrm{m}$ thickness; SigmaAldrich, Diegem, Belgium). The oven temperature program was as follows: initially $50^{\circ} \mathrm{C}$ for $2 \mathrm{~min}$, increasing by $5^{\circ} \mathrm{C} /$ min to $210^{\circ} \mathrm{C}$ and holding for $10 \mathrm{~min}$, followed by a second increase at $5^{\circ} \mathrm{C} / \mathrm{min}$ to $280^{\circ} \mathrm{C}$ and held at $280^{\circ} \mathrm{C}$ for $5 \mathrm{~min}$. Interface and ion source temperatures were both $250^{\circ} \mathrm{C}$ and the mass-to-charge ratio interval was 50-650 a.m.u. at 5.0 scans per second. Injections were carried out in splitless mode and helium $(1.2 \mathrm{~mL} / \mathrm{min})$ was used as carrier gas. The obtained electron impact ionization spectra were used to locate double bonds based on atomic mass unit distances with an interval of 12 a.m.u. between the most intense peaks of clusters of ions containing $n$ and $n-1$ carbon atoms, being interpreted as cleavage of the double bond between carbon $n$ and $n+1$ in the FA moiety. Besides this, the spectra were also used to locate hydroxyl groups based on comparisons with other reports and with the LipidWeb (www.lipidhome.co.uk).

Quantification of LCFA was based on the area of the internal standard and on the conversion of peak areas to the weight of FA by a theoretical response factor for each FA [56, 57].

For experiment 3 and 4, for each of the formed intermediates (i.e. cis-9, trans-11 CLA, trans-10, cis-12 CLA, trans-11 18:1 and trans-10 18:1) and the initial product (i.e. 18:2n-6), the proportion of total intermediates and initial product was calculated at each sampling time. This proportion was presented in relation to incubation time ( $\mathrm{x}$-axis). From this, the area under the curve was calculated over the $24 \mathrm{~h}$ of incubation (area units: \% of 
total intermediates and $18: 2 \mathrm{n}-6 \times$ hour). The average proportion over this $24 \mathrm{~h}$ incubation period was then calculated as the area under the curve divided by 24 . The average proportion should not be confused with the proportion after $12 \mathrm{~h}$ of incubation (= after half of the incubation period). In experiment 3 , this calculation was done for 18:2n-6 and its biohydrogenation intermediates for each treatment combination (bacterium $\times$ growth medium) to examine the effect of bacterial species and growth medium on the metabolism of 18:2n-6. In experiment 4 , the same calculation was done, but only for the control medium, to examine the effect of the biomass ratio of $B$. fibrisolvens to $C$. acnes in the inoculum on the metabolism of $18: 2 n-6$. To elucidate the influence of growth medium, the individual measured values for each FA after $24 \mathrm{~h}$ of incubation were used.

\section{Identification of unknown fatty acids by GC/MS}

DMOX derivatives were prepared from the FAME extracts in order to characterize unknown biohydrogenation intermediates by electron impact mass spectrometry. In total, 10 isomers were characterized by GC/MS (Fig. 4). The obtained mass spectra were compared with mass spectra from intermediates of $18: 3 n-3$ or $18: 2 n-6$ biohydrogenation
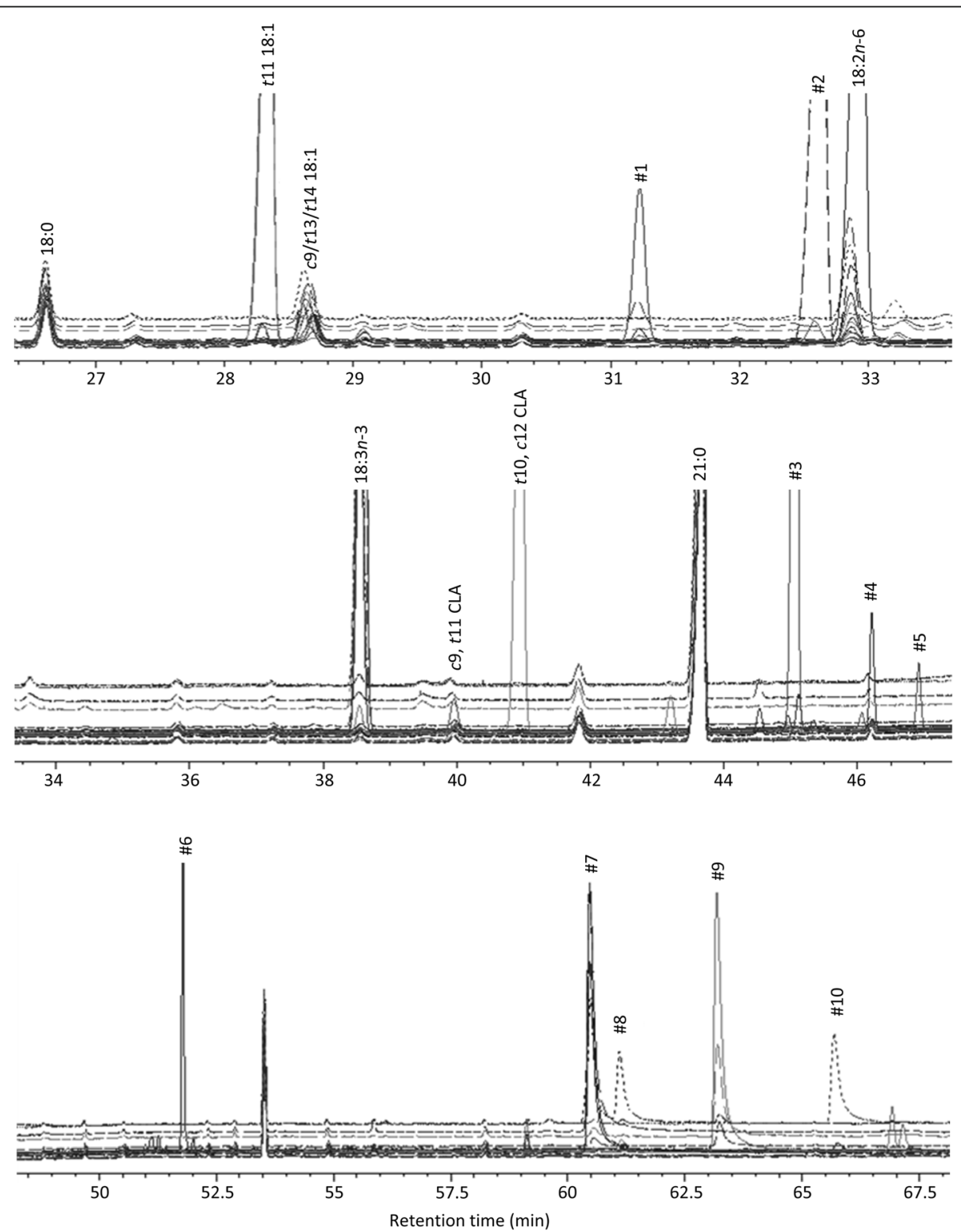

Fig. 4 Partial GC-MS chromatogram of the C18 FAME elution profile region. t, trans; C, cis; CLA, conjugated linoleic acid; \#1-\#10, unidentified compounds. Different chromatograms originate from incubations with distilled water or with pure cultures of Butyrivibrio fibrisolvens D1, Cutibacterium acnes DSM 1897, Streptococcus gallolyticus DSM 16831, Streptococcus equinus Pearl 11, Megasphaera elsdenii 2602A and 5052B under control growth conditions 
studies described in other reports (e.g. [29, 37, 38]) and with information from the LipidWeb (www.lipidhome.co. uk). In each mass spectrum with unknown isomers (Fig. 5), the base peak was observed at $\mathrm{m} / \mathrm{z} 113$, produced by a McLafferty arrangement. This peak was accompanied with a prominent ion at $m / z 126$, which is a characteristic ion in a DMOX derivative, and is formed by a cyclization displacement reaction.

The mass spectra of compound \#1 showed a gap of 12 a.m.u. at $m / z 196 / 208$ and at $m / z 250 / 262$. Therefore, this peak was identified as $\Delta 9,14-18: 2$. The double bond geometry could not be assigned through the used technique. The mass spectra of compound \#2 corresponded to the mass spectra of $\Delta 11,15-18: 2$ obtained in the experiment of Alves and Bessa [38], i.e. there was a gap of 12 a.m.u. at $m / z 224 / 236$ and at $m / z 278 / 290$. Since this isomer was produced when 18:3n-3 was incubated with $B$. fibrisolvens D1, which is an important trans-11 producer [6], we feel confident that the structure of $\Delta 11$,
15-18:2 is trans-11, cis-15 18:2, in accordance with Alves and Bessa [38]. Compounds \#3, \#4, \#5 and \#6 were identified in a similar way as cis-9, trans-11, cis-15 CLnA, trans-10, cis-12, cis-15 CLnA, $\Delta 9,11,15-18: 3$ and $\Delta 11,13,15-18: 3$, respectively [37].

Compound \#7 was identified as $13-\mathrm{OH} \Delta 9-18: 1$. The double bond was recognized by the gap of 12 a.m.u. at $m / z 196 / 208$, whereas the ions at $m / z 250$ and $m / z 280$ indicate the presence of a hydroxyl group at carbon atom 13. Compounds \#8, \#9 and \#10 were identified in a similar way as $10-\mathrm{OH} \Delta 12-18: 1,13-\mathrm{OH} \Delta 9,15-18: 2$ and 10-OH $\Delta 12,15-18: 2$, respectively.

\section{Statistical analysis}

Data were analyzed using the MIXED procedure of SAS (version Enterprise Guide 7.1; SAS Institute Inc., Cary, NC, US). In experiment 2 , the following model was used: $Y_{i j}=\mu+M_{i}+D_{j}+\varepsilon_{i j}$, with $M_{i}$ the fixed effect of growth medium $\left(i=\right.$ control or lactate-enriched medium), $D_{j}$ the
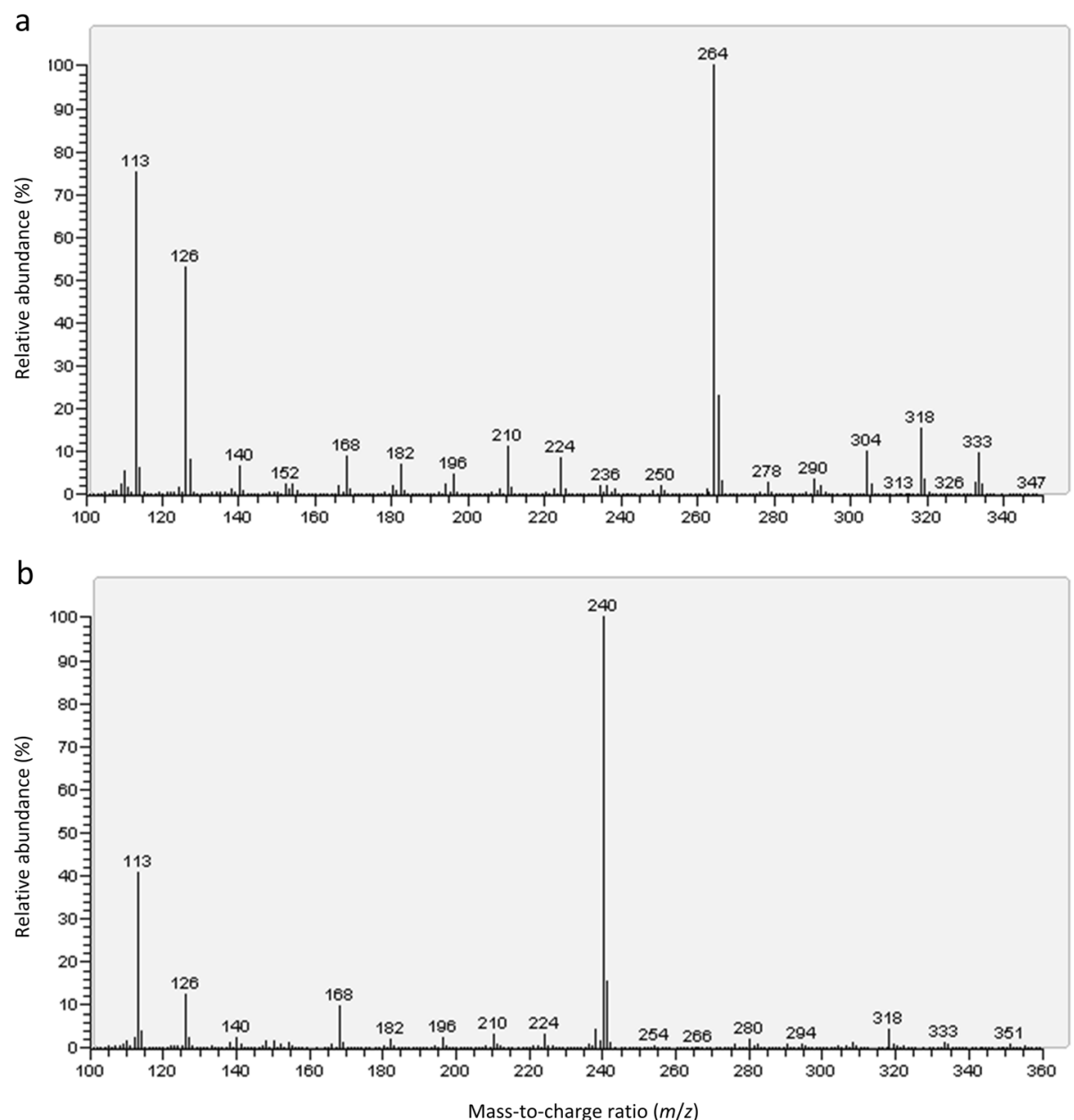

Fig. 5 Examples of electron impact mass spectra of DMOX derivatives. a, compound \#2, derived from pure culture incubation of Butyrivibrio fibrisolvens D1 under control growth conditions with $40 \mu \mathrm{g} / \mathrm{mL}$ 18:3n-3; b, compound \#8, derived from pure culture incubation of Megasphaera elsdenii 5052B under control growth conditions with $40 \mu \mathrm{g} / \mathrm{mL} 18: 2 \mathrm{n}-6$ 
random effect of day, and $\varepsilon_{i j}$ the residual error term. In experiment 3 and 4, the following model was used: $Y_{i j k}=$ $\mu+B_{i}+M_{j}+D_{k}+B_{i} \times M_{j}+\varepsilon_{i j k}$, with $B_{i}$ the fixed effect of bacterial species (exp. 3, $i=B$. fibrisolvens or $C$. acnes), or the fixed effect of the biomass ratio of $B$. fibrisolvens to $C$. acnes in the inoculum (exp. 4, $i=100 / 0,50 / 50,10 / 90,2 / 98$, $0.4 / 99.6$, or $0 / 100), M_{j}$ the fixed effect of growth medium ( $j$ = control, low $\mathrm{pH}$, or DHA-enriched medium), $D_{k}$ the random effect of day, and $\varepsilon_{i j k}$ the residual error term. Differences at $P<0.05$ were considered to be statistically significant and were evaluated by the Tukey-Kramer multiple comparison test.

\section{Supplementary information}

Supplementary information accompanies this paper at https://doi.org/10 1186/s12866-020-01876-7.

Additional file 1: Table S1. Influence of lactate on $\mathrm{OD}_{600}$ of different bacterial strains after $24 \mathrm{~h}$ of incubation with $40 \mu \mathrm{g} / \mathrm{mL}$ 18:2n-6 (Exp. 2). Table S2. Influence of lactate on volatile fatty acids produced by different bacterial strains after $24 \mathrm{~h}$ of incubation with $40 \mu \mathrm{g} / \mathrm{mL}$ 18:2n-6 (exp. 2). Table S3. Net production ( $\mu \mathrm{mol}$ per tube) of propionic acid and butyric acid by different biomass ratios of Butyrivibrio fibrisolvens D1 to Cutibacterium acnes DSM 1897 in the inoculum after $24 \mathrm{~h}$ of incubation under different growth conditions ${ }^{\mathrm{a}}$.

\section{Abbreviations}

CLA: Conjugated linoleic acid; CLnA: Conjugated linolenic acid; DHA: Docosahexaenic acid, 22:6n-3; DMOX: 4,4-dimethyloxazoline; FA: Fatty acid; FAME: Fatty acid methyl ester; LCFA: Long-chain fatty acid; OD: Optical density; VFA: Volatile fatty acid

\section{Acknowledgements}

The authors gratefully acknowledge Charlotte Melis for her assistance during the incubations and with the chemical analyses. Next to this, the authors also thank Dr. Paul Weimer and Dr. John Wallace for providing us bacteria from their laboratory, and as such, for giving us the opportunity to work with those bacteria. Finally, appreciation goes to Longhui Jing and Frederik Gadeyne for providing useful comments on the manuscript.

\section{Authors' contributions \\ VF, BV and LD conceived and designed the experiment. LD performed the experiments. LD and JJ analyzed the data. LD and JJ wrote the manuscript. $J J$ and VF revised the manuscript. All authors approved the submitted versions and agree to be accountable for all aspects of the work.}

\section{Funding}

The doctoral fellowship of LD is financed by the Special Research Fund of Ghent University (BOF, Bijzonder Onderzoeksfonds:BOF15/DOC/246).

\section{Availability of data and materials}

The datasets used and/or analyzed during the current study are available from the corresponding author on reasonable request.

\section{Ethics approval and consent to participate}

All procedures performed in studies involving animals were in accordance with the ethical standards of the Institute for Agricultural and Fisheries Research, Belgium. All applicable international, national, and/or institutional guidelines for the care and use of animals were followed. Fistulation of the sheep and cows was approved by the ethical commission of the Institute for Agricultural and Fisheries Research, Belgium (EC 2014, 241).

\section{Consent for publication}

Not applicable.

\section{Competing interests}

The authors declare that they have no competing interests.

\section{Author details}

${ }^{1}$ Laboratory for Animal Nutrition and Animal Product Quality (LANUPRO), Department of Animal Sciences and Aquatic Ecology, Ghent University, Ghent, Belgium. ${ }^{2}$ Present address: Research Group Marine Biology,

Department of Biology, Ghent University, Ghent, Belgium.

Received: 25 November 2019 Accepted: 25 June 2020

Published online: 07 July 2020

\section{References}

1. Ferlay A, Bernard L, Meynadier A, Malpuech-Brugère C. Production of trans and conjugated fatty acids in dairy ruminants and their putative effects on human health: a review. Biochimie. 2017;141:107-20.

2. Buccioni A, Decandia M, Minieri S, Molle G, Cabiddu A. Lipid metabolism in the rumen: new insights on lipolysis and biohydrogenation with an emphasis on the role of endogenous plant factors. Anim Feed Sci Technol. 2012;174:1-25.

3. Shingfield KJ, Wallace RJ. Chapter 1: synthesis of conjugated linoleic acid in ruminants and humans, in conjugated linoleic acids and conjugated vegetable oils; 2014. https://doi.org/10.1039/9781782620211-00001.

4. Bauman DE, Griinari JM. Nutritional regulation of milk fat synthesis. Annu Rev Nutr. 2003;23:203-27.

5. Harvatine KJ, Boisclair YR, Bauman DE. Recent advances in the regulation of milk fat synthesis. Animal. 2009;3:40-54

6. Kepler CR, Hirons KP, McNeill JJ, Tove SB. Intermediates and products of the biohydrogenation of linoleic acid by Butyrivibrio fibrisolvens. J Biol Chem. 1966:241:1350-4.

7. McKain N, Shingfield KJ, Wallace RJ. Metabolism of conjugated linoleic acids and 18:1 fatty acids by ruminal bacteria: products and mechanisms. Microbiol. 2010;156:579-88.

8. Maia MRG, Chaudhary LC, Figueres L, Wallace RJ. Metabolism of polyunsaturated fatty acids and their toxicity to the microflora of the rumen. Antonie Van Leeuwenhoek. 2007;91:303-14.

9. Paillard D, McKain N, Chaudhary LC, Walker ND, Pizette F, Koppova I, McEwan NR, Kopečný J, Vercoe PE, Louis P, Wallace RJ. Relation between phylogenetic position, lipid metabolism and butyrate production by different Butyrivibrio-like bacteria from the rumen. Antonie Van Leeuwenhoek. 2007;91:417-22.

10. Dewanckele L, Vlaeminck B, Fievez V. Sharpea azabuensis: a ruminal bacterium that produces trans-11 intermediates from linoleic and linolenic acid. Microbiolohy. 2019;165. https://doi.org/10.1099/mic.0.000811.

11. Gorissen L, Raes K, Weckx S, Dannenberger D, Leroy F, De Vuyst L, De Smet S. Production of conjugated linoleic acid and conjugated linolenic acid isomers by Bifidobacterium species. Appl Microbiol Biotechnol. 2010;87: 2257-66.

12. Renes E, Linares DM, González L, Fresno JM, Tornadijo ME, Stanton C. Study of the conjugated linoleic acid synthesis by Lactobacillus strains and by different co-cultures designed for this ability. J Funct Foods. 2017;35:74-80.

13. Mclntosh FM, Shingfield KJ, Devillard E, Russell WR, Wallace RJ. Mechanism of conjugated linoleic acid and vaccenic acid formation in human faecal suspensions and pure cultures of intestinal bacteria. Microbiol. 2009;155: 285-94.

14. Shingfield KJ, Kairenius P, Ärölä A, Paillard D, Muetzel S, Ahvenjärvi S, Vanhatalo A, Huhtanen P, Toivonen V, Griinari JM, Wallace RJ. Dietary fish oil supplements modify ruminal biohydrogenation, alter the flow of fatty acids at the omasum, and induce changes in the ruminal Butyrivibrio population in lactating cows. J Nutr. 2012;142:1437-48.

15. He M, Armentano LE. Effect of fatty acid profile in vegetable oils and antioxidant supplementation on dairy cattle performance and milk fat depression. J Dairy Sci. 2011;94:2481-91.

16. Wallace RJ, McKain N, Shingfield KJ, Devillard E. Isomers of conjugated linoleic acids are synthesized via different mechanisms in ruminal digesta and bacteria. J Lipid Res. 2007:48:2247-54.

17. Scholz CF, Kilian M. The natural history of cutaneous propionibacteria, and reclassification of selected species within the genus Propionibacterium to the proposed novel genera Acidipropionibacterium gen. nov., Cutibacterium gen. nov. and Pseudopropionibacterium gen. nov. Int I Syst Evol Microbiol. 2016;66:4422-32. 
18. Dewanckele L, Vlaeminck B, Hernandez-Sanabria E, Ruiz-González A, Debruyne S, Jeyanathan J, Fievez V. Rumen biohydrogenation and microbial community changes upon early life supplementation of 22:6n-3 enriched microalgae to goats. Front Microbiol. 2018;9:573.

19. Weimer PJ, Stevenson DM, Mertens DR. Shifts in bacterial community composition in the rumen of lactating dairy cows under milk fat-depressing conditions. J Dairy Sci. 2010;93:265-78.

20. Mohammed R, Stevenson DM, Beauchemin KA, Muck RE, Weimer PJ. Changes in ruminal bacterial community composition following feeding of alfalfa ensiled with a lactic acid bacterial inoculant. J Dairy Sci. 2012;95:328-39.

21. Dewanckele L, Jing L, Stefańska B, Vlaeminck B, Jeyanathan J, Van Straalen WM, Koopmans A, Fievez V. Distinct blood and milk 18-carbon fatty acid proportions and buccal bacterial populations in dairy cows differing in reticulorumen $\mathrm{pH}$ response to dietary supplementation of rapidly fermentable carbohydrates. J Dairy Sci. 2019;102:4025-40.

22. Kim YJ, Liu RH, Rychlik JL, Russell JB. The enrichment of a ruminal bacterium (Megasphaera elsdenii YJ-4) that produces the trans-10, cis-12 isomer of conjugated linoleic acid. J Appl Microbiol. 2002;92:976-82.

23. Kemp P, White RW, Lander DJ. The hydrogenation of unsaturated fatty acids by five bacterial isolates from the sheep rumen, including a new species. Microbiol. 1975;90:100-14.

24. Pitta DW, Indugu N, Vecchiarelli B, Rico DE, Harvatine KJ. Alterations in ruminal bacterial populations at induction and recovery from diet-induced milk fat depression in dairy cows. J Dairy Sci. 2018;101:295-309.

25. Zened A, Meynadier A, Cauquil L, Mariette J, Klopp C, Dejean S, Gonzalez I, Bouchez O, Enjalbert F, Combes S. Trans-11 to trans-10 shift of ruminal biohydrogenation of fatty acids is linked to changes in rumen microbiota. Gut Microbiol. 2016;Abstr. P-202:150 Proceedings of the 10th joint symposium on gut microbiology; 2016 June 20-23; Clermont-Ferrand, France.

26. Khafipour E, Li S, Plaizier JC, Krause DO. Rumen microbiome composition determined using two nutritional models of subacute ruminal acidosis. Appl Environ Microbiol. 2009;75:7115-24.

27. Hook SE, Steele MA, Northwood KS, Dijkstra J, France J, Wright ADG, McBride BW. Impact of subacute ruminal acidosis (SARA) adaptation and recovery on the density and diversity of bacteria in the rumen of dairy cows. FEMS Microbiol Ecol. 2011;78:275-84.

28. Plaizier JC, Krause DO, Gozho GN, McBride BW. Subacute ruminal acidosis in dairy cows: the physiological causes, incidence and consequences. Vet J. 2008; 176:21-31

29. Devillard E, McIntosh FM, Duncan SH, Wallace RJ. Metabolism of linoleic acid by human gut bacteria: different routes for biosynthesis of conjugated linoleic acid. J Bacteriol. 2007:189:2566-70.

30. Laverroux S, Glasser F, Gillet M, Joly C, Doreau M. Isomerization of vaccenic acid to cis and trans C18:1 isomers during biohydrogenation by rumen microbes. Lipids. 2011:46:843-50.

31. Hussain SKA, Srivastava A, Tyagi A, Shandilya UK, Kumar A, Kumar S, Panwar S, Tyagi AK. Characterization of CLA-producing Butyrivibrio spp. reveals strain-specific variations. 3. Biotech. 2016;6:90.

32. Fukuda S, Nakanishi Y, Chikayama E, Ohno H, Hino T, Kikuchi J. Evaluation and characterization of bacterial metabolic dynamics with a novel profiling technique, real-time metabolotyping. PLoS One. 2009;4:e4893. https://doi. org/10.1371/journal.pone.0004893.

33. Alonso L, Cuesta EP, Gilliland SE. Production of free conjugated linoleic acid by Lactobacillus acidophilus and Lactobacillus casei of human intestinal origin. J Dairy Sci. 2003:86:1941-6.

34. Hennessy AA, Barrett E, Ross RP, Fitzgerald GF, Devery R, Stanton C. The production of conjugated a-linolenic, $Y$-linolenic and stearidonic acids by strains of Bifidobacteria and Propionibacteria. Lipids. 2012;47:313-27.

35. Park HG, Cho HT, Song MC, Kim SB, Kwon EG, Choi NJ, Kim YJ. Production of a conjugated fatty acid by Bifidobacterium breve LMC520 from a-linolenic acid: conjugated linolenic acid (CLnA). J Agricultural Food Chem. 2012;60: 3204-10.

36. Maia MRG, Cabrita ARJ, Fonseca AJM, Wallace RJ. Biohydrogenation of alinolenic acid by the rumen bacterium Propionibacterium acnes. Gut Microbiol. 2016;Abstr. P-63:80 Proceedings of the 10th joint symposium on gut microbiology; 2016 June 20-23; Clermont-Ferrand, France.

37. Honkanen AM, Leskinen H, Toivonen V, McKain N, Wallace RJ, Shingfield KJ. Metabolism of a-linolenic acid during incubations with strained bovine rumen contents: products and mechanisms. Br J Nutr. 2016;115:2093-105.

38. Alves SP, Bessa RJB. The trans-10,cis-15 18:2: a missing intermediate of trans10 shifted rumen biohydrogenation pathway? Lipids. 2014;49:527-41.
39. Zened A, Troegeler-Meynadier A, Nicot MC, Combes S, Cauquil L, Farizon Y, et al. Starch and oil in the donor cow diet and starch in substrate differently affect the in vitro ruminal biohydrogenation of linoleic and linolenic acids. J Dairy Sci. 2011;94:5634-45.

40. Vlaeminck B, Khattab W, Fievez V. Is ruminal trans-11-18: 1 accumulation a prerequisite for trans-10-18: 1 production? Anim Prod Sci. 2015;55:225-30.

41. Chen L, Shen Y, Wang C, Ding L, Zhao F, Wang M, et al. Megasphaera elsdenii lactate degradation pattern shifts in rumen acidosis models. Front Microbiol. 2019;10:162.

42. Weimer PJ, Moen GN. Quantitative analysis of growth and volatile fatty acid production by the anaerobic ruminal bacterium Megasphaera elsdenii T81. Appl Microbiol Biotechnol. 2013;97:4075-81.

43. Lourenço M, Ramos-Morales E, Wallace RJ. The role of microbes in rumen lipolysis and biohydrogenation and their manipulation. Animal. 2010;4: 1008-23.

44. Choi NJ, Imm JY, Oh S, Kim BC, Hwang HJ, Kim YJ. Effect of pH and oxygen on conjugated linoleic acid (CLA) production by mixed rumen bacteria from cows fed high concentrate and high forage diets. Anim Feed Sci Technol. 2005;124:643-53.

45. Jiang J, Björck L, Fondén R. Production of conjugated linoleic acid by dairy starter cultures. J Appl Microbiol. 1998;85:95-102.

46. Kim YJ, Liu RH, Bond DR, Russell JB. Effect of linoleic acid concentration on conjugated linoleic acid production by Butyrivibrio fibrisolvens A38. Appl Environ Microbiol. 2000;66:5226-30.

47. Toral PG, Bernard L, Belenguer A, Rouel J, Hervás G, Chilliard Y, Frutos P. Comparison of ruminal lipid metabolism in dairy cows and goats fed diets supplemented with starch, plant oil, or fish oil. J Dairy Sci. 2016;99:301-16.

48. Zhu H, Fievez V, Mao S, He W, Zhu W. Dose and time response of ruminally infused algae on rumen fermentation characteristics, biohydrogenation and Butyrivibrio group bacteria in goats. J Anim Sci Biotechnol. 2016;7:22.

49. Boeckaert C, Fievez V, Van Hecke D, Verstraete W, Boon N. Changes in rumen biohydrogenation intermediates and ciliate protozoa diversity after algae supplementation to dairy cattle. Eur J Lipid Sci Technol. 2007;109:767-77.

50. Toral PG, Hervás $G$, Leskinen $H$, Shingfield $K$, Frutos P. In vitro ruminal biohydrogenation of eicosapentaenoic (EPA), docosapentaenoic (DPA), and docosahexaenoic acid (DHA) in cows and ewes: intermediate metabolites and pathways. J Dairy Sci. 2018;101:6109-21.

51. Zhao T, Ma Y, Qu Y, Luo H, Liu K, Zuo Z, Lu X. Effect of dietary oil sources on fatty acid composition of ruminal digesta and populations of specific bacteria involved in hydrogenation of 18-carbon unsaturated fatty acid in finishing lambs. Small Ruminant Res. 2016;144:126-34.

52. Harfoot CG, Noble RC, Moore JH. Food particles as a site for biohydrogenation of unsaturated fatty acids in the rumen. Biochem J. 1973; 132:829-32.

53. Jeyanathan J, Escobar M, Wallace RJ, Fievez V, Vlaeminck B. Biohydrogenation of 22:6n-3 by Butyrivibrio proteoclasticus P18. BMC Microbiol. 2016;16:104

54. Shingfield KJ, Bernard L, Leroux C, Chilliard Y. Role of trans fatty acids in the nutritional regulation of mammary lipogenesis in ruminants. Animal. 2010;4 1140-66.

55. Destaillats F, Trottier JP, Galvez JMG, Angers P. Analysis of a-linolenic acid biohydrogenation intermediates in milk fat with emphasis on conjugated linolenic acids. J Dairy Sci. 2005;88:3231-9.

56. Ackman RG, Sipos JC. Application of specific response factors in gas chromatographic analysis of methyl esters of fatty acids with flame ionization detectors. J Am Oil Chem Soc. 1964;41:377-8.

57. Wolff RL, Bayard CC, Fabien RJ. Evaluation of sequential methods for the determination of butterfat fatty acid composition with emphasis on trans18:1 acids. Application to the study of seasonal variations in French butters. J Am Oil Chem Soc. 1995;72:1471-83.

\section{Publisher's Note}

Springer Nature remains neutral with regard to jurisdictional claims in published maps and institutional affiliations. 